\title{
@creative
commons
}

ISSN 2590-9770

The Art of Discrete and Applied Mathematics 2 (2019) \#P2.07

https://doi.org/10.26493/2590-9770.1323.ceb

(Also available at http://adam-journal.eu)

\section{On noncommutative generalisations of Boolean algebras*}

\author{
Antonio Bucciarelli \\ Institut de Recherche en Informatique Fondamentale, \\ Université de Paris, 8 Place Aurélie Nemours, 75205 Paris Cedex 13, France \\ Antonino Salibra ${ }^{\dagger}$ \\ Department of Environmental Sciences, Informatics and Statistics, \\ Università Ca' Foscari Venezia, Via Torino 155, 30173 Venezia, Italia
}

Received 22 August 2019, accepted 7 December 2019, published online 30 December 2019

\begin{abstract}
Skew Boolean algebras (SBA) and Boolean-like algebras ( $n \mathrm{BA})$ are one-pointed and $n$-pointed noncommutative generalisation of Boolean algebras, respectively. We show that any $n \mathrm{BA}$ is a cluster of $n$ isomorphic right-handed SBAs, axiomatised here as the variety of skew star algebras. The variety of skew star algebras is shown to be term equivalent to the variety of $n$ BAs. We use SBAs in order to develop a general theory of multideals for $n$ BAs. We also provide a representation theorem for right-handed SBAs in terms of $n \mathrm{BAs}$ of $n$-partitions.
\end{abstract}

Keywords: Skew Boolean algebras, Boolean-like algebras, Church algebras, multideals.

Math. Subj. Class.: 06E75, 03G05, 08B05, 08A30

\section{Introduction}

Boolean algebras are the main example of a well-behaved double-pointed variety - meaning a variety $\mathcal{V}$ whose type includes two distinct constants 0,1 in every nontrivial $\mathbf{A} \in \mathcal{V}$. Since there are other double-pointed varieties of algebras that have Boolean-like features, in $[15,23]$ the notion of Boolean-like algebra (of dimension 2) was introduced as a generalisation of Boolean algebras to a double-pointed but otherwise arbitrary similarity type.

\footnotetext{
${ }^{*}$ The results of this paper were presented at Non-commutative structures 2018: A workshop in honor of Jonathan Leech. The authors would like to thank the anonymous reviewer for the constructive comments, which helped us to improve the presentation of the paper.

${ }^{\dagger}$ Corresponding author.

E-mail addresses: buccia@irif.fr (Antonio Bucciarelli), salibra@unive.it (Antonino Salibra)
} 
The idea behind this approach was that a Boolean-like algebra of dimension 2 is an algebra A such that every $a \in A$ is 2-central in the sense of Vaggione [27], meaning that $\theta(a, 0)$ and $\theta(a, 1)$ are complementary factor congruences of $\mathbf{A}$. Central elements can be given an equational characterisation through the ternary operator $q$ satisfying the fundamental properties of the if-then-else connective of computer science. Algebraic analogues of the if-then-else construction have been studied extensively in the literature; the best known of these realisations is the ternary discriminator function $t: A^{3} \rightarrow A$ of general algebra [28], defined for all $a, b, c \in A$ by $t(a, b, c)=c$ if $a=b$ and $a$ otherwise. Varieties generated by a class of algebras with a common discriminator term are called discriminator varieties and are the most successful generalisation of Boolean algebras to date ([7, Section IV.9]).

It turns out that some important properties of Boolean algebras are shared by $n$-pointed algebras whose elements satisfy all the equational conditions of $n$-central elements through an operator $q$ of arity $n+1$ satisfying the fundamental properties of a generalised if-thenelse connective. These algebras, and the varieties they form, were termed Boolean-like algebras of dimension $n$ ( $n \mathrm{BA}$, for short) in [6]. Varieties of $n \mathrm{BAs}$ have many remarkable properties in common with the variety of Boolean algebras. In particular, any variety of $n$ BAs with compatible operations is generated by the $n$ BAs of finite cardinality $n$. In the pure case (i.e., when the type includes just the generalised if-then-else $q$ and the $n$ constants $\mathrm{e}_{1}, \ldots, \mathrm{e}_{n}$ ), there is (up to isomorphism) a unique $n$ BA $\mathbf{n}$ of cardinality $n$, so that any pure $n \mathrm{BA}$ is isomorphic to a subalgebra of $\mathbf{n}^{I}$, for a suitable set $I$. Another remarkable property of the 2-element Boolean algebra is the definability of all finite Boolean functions in terms of the connectives AND, OR, NOT. This property is inherited by the algebra n: all finite functions on the universe of $\mathbf{n}$ are term-definable, so that the variety of pure $n$ BAs is primal. More generally, a variety of an arbitrary type with one generator is primal if and only if it is a variety of $n$ BAs.

Lattices and boolean algebras have been generalised in other directions: in the last decades weakenings of lattices where the meet and join operations may fail to be commutative have attracted the attention of various researchers. A non-commutative generalisation of lattices, probably the most interesting and successful, is the concept of skew lattice [16] along with the related notion of skew Boolean algebra (SBA) (the interested reader is referred to [3, 17, 18] or [26] for a comprehensive account). Here, a SBA is a symmetric skew lattice with zero in the sense of Leech [16], structurally enriched with an implicative BCS-difference [4] operation. Roughly speaking, a SBA is a non-commutative analogue of a generalised Boolean algebra. The significance of SBAs is revealed by a result of Leech [17], stating that any right-handed SBA can be embedded into some SBA of partial functions. This result has been revisited and further explored in [1] and [14], showing that any SBA is dual to a sheaf over a locally-compact Boolean space.

SBAs are also closely related to discriminator varieties (see [3, 9] for the one-pointed case and [23] for the double-pointed one). Seminal results of Bignall and Leech [3] show that every algebra in a one-pointed discriminator variety can be presented, up to term equivalence, as a skew Boolean intersection algebra (SBIA) with compatible operations. SBIAs are closely related to the SBAs of Leech [17]. Every SBIA has a SBA term reduct, but not conversely.

The present paper explores the connection between skew Boolean algebras and Booleanlike algebras of dimension $n$. We prove that any $n \mathrm{BA} \mathbf{A}$ contains a symmetric $\cap$-skew cluster of right-handed SBIAs $S_{1}^{\cap}(\mathbf{A}), \ldots, S_{n}^{\cap}(\mathbf{A})$, called its $\cap$-skew reducts. Interestingly, 
every permutation $\sigma$ of the symmetric group $S_{n}$ determines a bunch of isomorphisms

$$
\mathrm{S}_{1}^{\cap}(\mathbf{A}) \cong \mathrm{S}_{\sigma 1}^{\cap}(\mathbf{A}), \ldots, \mathrm{S}_{n}^{\cap}(\mathbf{A}) \cong \mathrm{S}_{\sigma n}^{\cap}(\mathbf{A})
$$

which shows the inner symmetry of the $n$ BAs. Every $n$ BA has also a skew cluster $S_{1}(\mathbf{A}), \ldots, S_{n}(\mathbf{A})$ of isomorphic right-handed SBAs, called its skew reducts, which are the skew Boolean algebra reducts of members of the $\cap$-skew cluster of $\mathbf{A}$. The skew reducts of a $n \mathrm{BA}$ are so deeply correlated that they allow us to recover the full structure of the $n$ BA. We introduce a new variety of algebras, called skew star algebras, equationally axiomatising a bunch of skew Boolean algebras and their relationships, and we prove that it is term equivalent to the variety of $n \mathrm{BAs}$. We also provide a representation theorem for right-handed skew Boolean algebras in terms of $n \mathrm{BAs}$ of $n$-partitions. This result follows on combining Leech's example [17] showing that every right-handed skew Boolean algebra can be embedded in an algebra of partial functions with codomain $\{1,2\}$ with the result given in [6] that every $n \mathrm{BA}$ is isomorphic to a $n \mathrm{BA}$ of $n$-partitions.

The notion of ideal plays an important role in order theory and universal algebra. Ideals, filters and congruences are interdefinable in Boolean algebras. In the case of $n \mathrm{BAs}$, the couple ideal-filter is replaced by multideals, which are tuples $\left(I_{1}, \ldots, I_{n}\right)$ of disjoint skew Boolean ideals satisfying some compatibility conditions that extend in a conservative way those of the Boolean case. We show that there exists a bijective correspondence between multideals and congruences on $n \mathrm{BAs}$, rephrasing the well known correspondence of the Boolean case. The proof of this result makes an essential use of the notion of a coordinate, originally defined in [6] and rephrased here in terms of the operations of the skew reducts. Any element $a$ of a $n \mathrm{BA} \mathbf{A}$ univocally determines a $n$-tuple of elements of the canonical inner Boolean algebra $\mathbf{B}$ of $\mathbf{A}$, its coordinates, codifying $a$ as a "linear combination". In the Boolean case, there is a bijective correspondence between maximal ideals and homomorphisms onto 2 . In the last section of the paper we show that every multideal can be extended to an ultramultideal, and that there exists a bijective correspondence between ultramultideals and homomorphisms onto $\mathbf{n}$. Moreover, ultramultideals are proved to be exactly the prime multideals.

\section{Preliminaries}

The notation and terminology in this paper are pretty standard. For concepts, notations and results not covered hereafter, the reader is referred to [7,21] for universal algebra, to $[17,18,26]$ for skew Boolean algebras and to [6, 15, 23] for $n$ BAs.

\subsection{Algebras}

If $\tau$ is an algebraic type, an algebra $\mathbf{A}$ of type $\tau$ is called $a \tau$-algebra, or simply an algebra when $\tau$ is clear from the context. An algebra is trivial if its carrier set is a singleton set.

Superscripts that mark the difference between operations and operation symbols will be dropped whenever the context is sufficient for a disambiguation.

$\operatorname{Con}(\mathbf{A})$ is the lattice of all congruences on $\mathbf{A}$, whose bottom and top elements are, respectively, $\Delta=\{(a, a): a \in A\}$ and $\nabla=A \times A$. Given $a, b \in A$, we write $\theta(a, b)$ for the smallest congruence $\theta$ such that $(a, b) \in \theta$.

We say that an algebra $\mathbf{A}$ is:

(i) subdirectly irreducible if the lattice $\operatorname{Con}(\mathbf{A})$ has a unique atom; 
(ii) simple if $\operatorname{Con}(\mathbf{A})=\{\Delta, \nabla\}$;

(iii) directly indecomposable if $\mathbf{A}$ is not isomorphic to a direct product of two nontrivial algebras.

A class $\mathcal{V}$ of $\tau$-algebras is a variety (equational class) if it is closed under subalgebras, direct products and homomorphic images. If $K$ is a class of $\tau$-algebras, the variety $\mathcal{V}(K)$ generated by $K$ is the smallest variety including $K$. If $K=\{\mathbf{A}\}$ we write $\mathcal{V}(\mathbf{A})$ for $\mathcal{V}(\{\mathbf{A}\})$.

Following Blok and Pigozzi [5], two elements $a, b$ of an algebra $\mathbf{A}$ are said to be residually distinct if they have distinct images in every non-trivial homomorphic image of $\mathbf{A}$.

We say that a variety $\mathcal{V}$ is $n$-pointed iff it has at least $n$ nullary operators that are residually distinct in any nontrivial member of $\mathcal{V}$. Boolean algebras are the main example of a double-pointed variety.

A one-pointed variety $\mathcal{V}$ is 0 -regular if the congruences of algebras in $\mathcal{V}$ are uniquely determined by their 0 -classes. Fichtner [10] has shown that a one-pointed variety is 0 regular if and only if there exist binary terms $d_{1}(x, y), \ldots, d_{n}(x, y)$ satisfying the following two conditions:

- $d_{i}(x, x)=0$ for every $i=1, \ldots, n$;

- $d_{1}(x, y)=d_{2}(x, y)=\cdots=d_{n}(x, y)=0 \Rightarrow x=y$.

\subsubsection{Notations}

If $A$ is a set and $X \subseteq A$, then $\bar{X}$ denotes the set $A \backslash X$.

Let $\hat{n}=\{1, \ldots, n\}$ and $q$ be an operator of arity $n+1$. If $d_{1}, \ldots, d_{k}$ is a partition of $\hat{n}$ and $a, b_{1} \ldots, b_{k} \in A$, then

$$
q\left(a, b_{1} / d_{1}, \ldots, b_{k} / d_{k}\right)
$$

denotes $q\left(a, c_{1}, \ldots, c_{n}\right)$, where for all $1 \leq i \leq n, c_{i}=b_{j}$ iff $i \in d_{j}$. Notice that $q\left(a, b_{1} / d_{1}, \ldots, b_{k} / d_{k}\right)$ is well-defined as $d_{1}, \ldots, d_{k}$ partition $\hat{n}$. If $d_{j}$ is a singleton $\{i\}$, then we write $b / i$ for $b / d_{j}$. If $d_{i}=\hat{n} \backslash d_{r}$ is the complement of $d_{r}$, then we may write $b / \bar{d}_{r}$ for $b / d_{i}$. The notation (2.1) will be used extensively throughout the paper, mainly to define derived term operations in the context of $n \mathrm{BAs}$.

\subsection{Factor congruences and decomposition}

Directly indecomposable algebras play an important role in the characterisation of the structure of a variety of algebras. For example, if the class of indecomposable algebras in a Church variety (see Section 3.1 and [23]) is universal, then any algebra in the variety is a weak Boolean product of directly indecomposable algebras. In this section we summarize the basic ingredients of factorisation: tuples of complementary factor congruences and decomposition operators (see [21]).

Definition 2.1. A sequence $\left(\phi_{1}, \ldots, \phi_{n}\right)$ of congruences on a $\tau$-algebra $\mathbf{A}$ is a $n$-tuple of complementary factor congruences exactly when:

(1) $\bigcap_{1 \leq i \leq n} \phi_{i}=\Delta$;

(2) $\forall\left(a_{1}, \ldots, a_{n}\right) \in A^{n}$, there is $u \in A$ such that $a_{i} \phi_{i} u$, for all $1 \leq i \leq n$. 
Such an element $u$ such that $a_{i} \phi_{i} u$ for every $i$ is unique by Definition 2.1(1).

If $\left(\phi_{1}, \ldots, \phi_{n}\right)$ is a $n$-tuple of complementary factor congruences on $\mathbf{A}$, then the function $f: \mathbf{A} \rightarrow \prod_{i=1}^{n} \mathbf{A} / \phi_{i}$, defined by $f(a)=\left(a / \phi_{1}, \ldots, a / \phi_{n}\right)$, is an isomorphism. Moreover, every factorisation of $\mathbf{A}$ in $n$ factors univocally determines a $n$-tuple of complementary factor congruences.

A pair $\left(\phi_{1}, \phi_{2}\right)$ of congruences is a pair of complementary factor congruences if and only if $\phi_{1} \cap \phi_{2}=\Delta$ and $\phi_{1} \circ \phi_{2}=\nabla$. The pair $(\Delta, \nabla)$ corresponds to the product $\mathbf{A} \cong \mathbf{A} \times \mathbf{1}$, where $\mathbf{1}$ is a trivial algebra; obviously $\mathbf{1} \cong \mathbf{A} / \nabla$ and $\mathbf{A} \cong \mathbf{A} / \Delta$.

A factor congruence is any congruence which belongs to a pair of complementary factor congruences. The set of factor congruences of $\mathbf{A}$ is not, in general, a sublattice of $\operatorname{Con}(\mathbf{A})$.

Notice that, if $\left(\phi_{1}, \ldots, \phi_{n}\right)$ is a $n$-tuple of complementary factor congruences, then $\phi_{i}$ is a factor congruence for each $1 \leq i \leq n$, because the pair $\left(\phi_{i}, \bigcap_{j \neq i} \phi_{j}\right)$ is a pair of complementary factor congruences.

It is possible to characterise $n$-tuples of complementary factor congruences in terms of certain algebra homomorphisms called decomposition operators (see [21, Definition 4.32] for additional details).

Definition 2.2. An $n$-ary decomposition operator on a $\tau$-algebra $\mathbf{A}$ is a function $f: A^{n} \rightarrow$ $A$ satisfying the following conditions:

(D1) $f(x, x, \ldots, x)=x$;

(D2) $f\left(f\left(x_{11}, x_{12}, \ldots, x_{1 n}\right), \ldots, f\left(x_{n 1}, x_{n 2}, \ldots, x_{n n}\right)\right)=f\left(x_{11}, \ldots, x_{n n}\right)$;

(D3) $f$ is an algebra homomorphism from $\mathbf{A}^{n}$ to $\mathbf{A}$ :

$$
\begin{aligned}
f\left(g\left(x_{11}, x_{12}, \ldots, x_{1 k}\right), \ldots,\right. & \left.g\left(x_{n 1}, x_{n 2}, \ldots, x_{n k}\right)\right) \\
& =g\left(f\left(x_{11}, \ldots, x_{n 1}\right), \ldots, f\left(x_{1 k}, \ldots, x_{n k}\right)\right),
\end{aligned}
$$

for every $g \in \tau$ of arity $k$.

There is a bijective correspondence between $n$-tuples of complementary factor congruences and $n$-ary decomposition operators, and thus, between $n$-ary decomposition operators and factorisations of an algebra in $n$ factors.

Theorem 2.3. Any n-ary decomposition operator $f: \mathbf{A}^{n} \rightarrow \mathbf{A}$ on an algebra $\mathbf{A}$ induces a n-tuple of complementary factor congruences $\phi_{1}, \ldots, \phi_{n}$, where each $\phi_{i} \subseteq A \times A$ is defined by:

$$
\left.a \phi_{i} b \quad \text { iff } \quad f(a, \ldots, a, b, a, \ldots, a)=a \quad \text { (b at position } i\right) .
$$

Conversely, any n-tuple $\phi_{1}, \ldots, \phi_{n}$ of complementary factor congruences induces a decomposition operator $f$ on $\mathbf{A}: f\left(a_{1}, \ldots, a_{n}\right)=u$ iff $a_{i} \phi_{i} u$ for all $i$.

We say that two functions $f: A^{m} \rightarrow A$ and $g: A^{n} \rightarrow A$ commute (see [21, Definition 4.34]) if

$$
\begin{aligned}
f\left(g\left(x_{11}, \ldots, x_{1 n}\right), \ldots, g\left(x_{m 1}, \ldots, x_{m n}\right)\right) & \\
& =g\left(f\left(x_{11}, \ldots, x_{m 1}\right), \ldots, f\left(x_{1 n}, \ldots, x_{m n}\right)\right) .
\end{aligned}
$$

In this case, $f$ is a homomorphism from $(A, g)^{m}$ into $(A, g)$ and $g$ is a homomorphism from $(A, f)^{n}$ into $(A, f)$.

The following proposition is [21, Exercise 4.38(15)]. 
Proposition 2.4. Let $f$ and $g$ be an $m$-ary and an $n$-ary decomposition operator of an algebra A. Then $f\left(g\left(x_{11}, \ldots, x_{1 n}\right), \ldots, g\left(x_{m 1}, \ldots, x_{m n}\right)\right)$ is a decomposition operator of $\mathbf{A}$ if and only if $f$ and $g$ commute.

The variables occurring in $f\left(g\left(x_{11}, \ldots, x_{1 n}\right), \ldots, g\left(x_{m 1}, \ldots, x_{m n}\right)\right)$ may not all be distinct, as explained in the following proposition.

Proposition 2.5. If $f$ is a n-ary decomposition operator and $d_{1}, \ldots, d_{k}(k \geq 2)$ is a partition of $\hat{n}=\{1, \ldots, n\}$, then the map $h$, defined by

$$
h\left(y_{1}, \ldots, y_{k}\right)=f\left(z_{1}, \ldots, z_{n}\right) \text {, where for all } 1 \leq i \leq n, z_{i}=y_{j} \text { iff } i \in d_{j},
$$

is a k-ary decomposition operator.

\subsection{Factor elements}

The notion of decomposition operator and of factorisation can sometimes be internalised: some elements of the algebra, the so called factor elements, can embody all the information codified by a decompostion operator.

Let $\mathbf{A}$ be a $\tau$-algebra, where we distinguish a $(n+1)$-ary term operation $q$.

Definition 2.6. We say that an element $e$ of $\mathbf{A}$ is a factor element with respect to $q$ if the $n$-ary operation $f_{e}: A^{n} \rightarrow A$, defined by

$$
f_{e}\left(a_{1}, \ldots, a_{n}\right)=q^{\mathbf{A}}\left(e, a_{1}, \ldots, a_{n}\right), \text { for all } a_{i} \in A,
$$

is a $n$-ary decomposition operator (that is, $f_{e}$ satisfies identities (D1)-(D3) of Definition 2.2).

An element $e$ of $\mathbf{A}$ is a factor element if and only if the tuple of relations $\left(\phi_{1}, \ldots, \phi_{n}\right)$, defined by $a \phi_{i} b$ iff $q(e, a, \ldots, a, b, a, \ldots, a)=a$ ( $b$ at position $\left.i\right)$, constitute a $n$-tuple of complementary factor congruences of $\mathbf{A}$.

By [9, Proposition 3.4] the set of factor elements is closed under the operation $q$ : if $a, b_{1}, \ldots, b_{n} \in A$ are factor elements, then $q\left(a, b_{1}, \ldots, b_{n}\right)$ is also a factor element.

We notice that

- different factor elements may define the same tuple of complementary factor congruences;

- there may exist $n$-tuples of complementary factor congruences that do not correspond to any factor element.

In Section 3 we describe a class of algebras, called Church algebras of dimension $n$, where the $(n+1)$-ary operator $q$ induces a bijective correspondence between a suitable subset of factor elements, the so-called $n$-central elements, and the set of all $n$-ary decomposition operators.

\subsection{Skew Boolean algebras}

We review here some basic definitions and results on skew lattices [16] and skew Boolean algebras [17]. 
Definition 2.7. A skew lattice is an algebra $\mathbf{A}=(A, \vee, \wedge)$ of type $(2,2)$, where both $\vee$ and $\wedge$ are associative, idempotent binary operations, connected by the absorption laws: $x \vee(x \wedge y)=x=x \wedge(x \vee y)$; and $(y \wedge x) \vee x=x=(y \vee x) \wedge x$.

The absorption conditions are equivalent to the following pair of biconditionals: $a \vee b=$ $b$ iff $a \wedge b=a$; and $a \vee b=a$ iff $a \wedge b=b$.

In any skew lattice we define the following relations:

1. $a \leq b$ iff $a \wedge b=a=b \wedge a$.

2. $a \preceq_{\mathcal{D}} b$ iff $a \wedge b \wedge a=a$.

3. $a \preceq_{\mathcal{L}} b$ iff $a \wedge b=a$.

4. $a \preceq_{\mathcal{R}} b$ iff $b \wedge a=a$.

The relation $\leq$ is a partial ordering, while the relations $\preceq_{\mathcal{D}}, \preceq_{\mathcal{L}}, \preceq_{\mathcal{R}}$ are preorders. The equivalences $\mathcal{D}, \mathcal{L}$ and $\mathcal{R}$, respectively induced by $\preceq_{\mathcal{D}}, \preceq_{\mathcal{L}}$ and $\preceq_{\mathcal{R}}$, are congruences. For more details see Schein [24].

A skew lattice is right-handed (left-handed) if $\mathcal{R}=\mathcal{D}(\mathcal{L}=\mathcal{D})$. The following conditions are equivalent for a skew lattice $\mathbf{A}$ :

(a) $\mathbf{A}$ is right-handed (left-handed);

(b) for all $a, b \in A, a \wedge b \wedge a=b \wedge a(a \wedge b \wedge a=a \wedge b)$.

Observe that

(i) The quotient $\mathbf{A} / \mathcal{D}$ is the maximal lattice image of $\mathbf{A}$. This is the skew-lattice theoretic analogue [16, Theorem 1.7] of the well-known Clifford-McLean theorem for bands.

(ii) The algebras $\mathbf{A} / \mathcal{L}$ and $\mathbf{A} / \mathcal{R}$ are the maximal right-handed and left-handed images of $\mathbf{A}$ respectively.

(iii) The skew lattice $\mathbf{A}$ is the fibered product of its maximal right-handed image $\mathbf{A} / \mathcal{L}$ with its maximal left-handed $\mathbf{A} / \mathcal{R}$ over its maximal lattice image. This result is the skew-lattice theoretic analogue [16, Theorem 1.15] of the Kimura factorisation theorem for idempotent semigroups.

In a skew lattice elements commuting under $\vee$ need not commute under $\wedge$ and viceversa. A skew lattice, satisfying $x \wedge y=y \wedge x$ if and only if $x \vee y=y \vee x$ for all $x$ and $y$, is called symmetric. Symmetric skew lattices form a variety characterised by the following identities (see [25, Theorem SSL-6]):

$$
x \vee y \vee(x \wedge y)=(y \wedge x) \vee y \vee x ; \quad x \wedge y \wedge(x \vee y)=(y \vee x) \wedge y \wedge x
$$

The two most significant classes of examples, skew lattices of idempotents in rings (see, e.g., [16]) and skew Boolean algebras (see [17] and Definition 2.8 below), consist of symmetric skew lattices.

If we expand skew lattices by a subtraction operation and a constant 0 , we get the following non-commutative variant of Boolean algebras (see [17]). 
Definition 2.8. A skew Boolean algebra (SBA, for short) is an algebra $\mathbf{A}=(A, \vee, \wedge, \backslash, 0)$ of type $(2,2,2,0)$ such that:

(S1) its reduct $(A, \vee, \wedge)$ is a skew lattice satisfying

- Normality: $x \wedge y \wedge z \wedge x=x \wedge z \wedge y \wedge x$;

- Symmetry: $x \wedge y=y \wedge x$ iff $x \vee y=y \vee x$;

- Distributivity: $x \wedge(y \vee z) \wedge x=(x \wedge y \wedge x) \vee(x \wedge z \wedge x)$ and $x \vee(y \wedge z) \vee x=$ $(x \vee y \vee x) \wedge(x \vee z \vee x)$

(S2) 0 is left and right absorbing w.r.t. skew lattice meet;

(S3) the operation \satisfies the identities

$$
\begin{aligned}
& (x \wedge y \wedge x) \vee(x \backslash y)=x=(x \backslash y) \vee(x \wedge y \wedge x) \\
& x \wedge y \wedge x \wedge(x \backslash y)=0=(x \backslash y) \wedge x \wedge y \wedge x
\end{aligned}
$$

Every SBA is strongly distributive, i.e., it satisfies the identities $x \wedge(y \vee z)=(x \wedge y) \vee$ $(x \wedge z)$ and $(y \vee z) \wedge x=(y \wedge x) \vee(z \wedge x)$.

It can be seen that, for every $a \in A$, the natural partial order of the subalgebra $a \wedge A \wedge$ $a=\{a \wedge b \wedge a: b \in A\}=\{b: b \leq a\}$ of $\mathbf{A}$ is a Boolean lattice. Indeed, the algebra $(a \wedge A \wedge a, \vee, \wedge, 0, a, \neg)$, where $\neg b=a \backslash b$ for every $b \leq a$, is a Boolean algebra with minimum 0 and maximum $a$.

Notice that

- The normal axiom implies the commutativity of $\wedge$ and $\vee$ in the interval $a \wedge A \wedge a$.

- Axiom (S2) expresses that 0 is the minimum of the natural partial order on $A$.

- Axiom (S3) implies that, for every $b \in a \wedge A \wedge a$, the element $a \backslash b$ is the complement of $b$ in the Boolean lattice $a \wedge A \wedge a$. We point out here that $a \backslash b$ is in fact a kind of relative complement that acts 'locally' on subalgebras of the form $a \wedge A \wedge a$.

An element $m$ of a SBA $\mathbf{A}$ is maximal if $a \preceq \mathcal{D} m$ for every $a \in A$ (i.e., $a \wedge m \wedge a=a$, for every $a \in A$ ). When they exist, maximal elements form an equivalence class (modulo $\mathcal{D})$ called the maximal class. If $\mathbf{A}$ is a SBA, then $\mathbf{A} / \mathcal{D}$, where $\mathcal{D}$ is the Clifford-McLean congruence on $\mathbf{A}$, is a Boolean algebra iff $\mathbf{A}$ has a maximal class. Skew Boolean algebras with a maximal class thus constitute a very specialised class of skew Boolean algebras. It is known that every skew Boolean algebra embeds into a skew Boolean algebra with a maximal class.

A nonempty subset $I$ of a SBA A closed under $\vee$ is an ideal of A (see [19, Section 4]) if it satisfies one of the following equivalent conditions:

- $a \in A, b \in I$ and $a \preceq_{\mathcal{D}} b$ imply $a \in I$;

- $a \in A$ and $b \in I$ imply $a \wedge b, b \wedge a \in I$;

- $a \in A$ and $b \in I$ imply $a \wedge b \wedge a \in I$.

Given a congruence $\phi$ on a SBA, the equivalence class $0 / \phi$ is an ideal. However, congruences on a SBA are not in general in 1-1 correspondence with ideals. In particular, the congruence lattices of SBAs may satisfy no special lattice identities and they need not be congruence $n$-permutable for any $n \geq 2$. 


\subsection{Skew Boolean algebras with intersections}

Skew Boolean algebras such that every finite subset of their universe has an infimum w.r.t. the underlying natural partial ordering of the algebra stand out for their significance. We denote the infimum of $a$ and $b$ w.r.t. the natural partial order by $a \cap b$ and refer to the operation $\cap$ as intersection in order to distinguish it from the skew lattice meet $\wedge$. It turns out that SBAs augmented with the additional operation $\cap$ can be given an equational characterisation provided we include the operation $\cap$ into the signature.

Definition 2.9. A skew Boolean $\cap$-algebra (SBIA, for short) is an algebra $\mathbf{A}=(A ; \vee, \wedge$, $\cap, \backslash, 0)$ of type $(2,2,2,2,0)$ such that:

(i) The reduct $(A ; \vee, \wedge, \backslash, 0)$ is a SBA and the reduct $(A ; \cap)$ is a meet semilattice;

(ii) A satisfies the identities $x \cap(x \wedge y \wedge x)=x \wedge y \wedge x$ and $x \wedge(x \cap y)=x \cap y=$ $(x \cap y) \wedge x$.

The next theorem by Bignall and Leech [3], which we present in its simplest form, provides a powerful bridge between the theories of SBAs and pointed discriminator varieties.

Theorem 2.10. The variety of type $(3,0)$ generated by the class of all one-pointed discriminator algebras $(A ; t, 0)$, where $t$ is the discriminator function on $A$ and 0 is a constant, is term equivalent to the variety of right handed SBIAs.

\subsection{A term equivalence result for skew Boolean algebras}

In [9] Cvetko-Vah and the second author have introduced the variety of semicentral right Church algebras (SRCAs) and have shown that the variety of right-handed SBAs is term equivalent to the variety of SRCAs. It is worth noticing that, in SRCAs, a single ternary operator $q$ replaces all the binary operators of SBAs.

An algebra $\mathbf{A}=(A, q, 0)$ of type $(3,0)$ is called a right Church algebra (RCA, for short) if it satisfies the identity $q(0, x, y)=y$.

Definition 2.11. Let $\mathbf{A}=(A, q, 0)$ be a RCA. An element $a \in A$ is called semicentral if it is a factor element (w.r.t. $q$ ) satisfying $q(a, a, 0)=a$.

Lemma 2.12 ([9, Proposition 3.9]). Let $\mathbf{A}=(A, q, 0)$ be an RCA. Every semicentral element $e \in A$ determines a pair of complementary factor congruences:

$$
\phi_{e}=\{(a, b): q(e, a, b)=a\} \quad \text { and } \quad \bar{\phi}_{e}=\{(a, b): q(e, a, b)=b\}
$$

such that $\phi_{e}=\theta(e, 0)$, the least congruence of $\mathbf{A}$ equating e and 0.

Definition 2.13. An algebra $\mathbf{A}=(A, q, 0)$ of type $(3,0)$ is called a semicentral $\mathrm{RCA}$ (SRCA, for short) if every element of $A$ is semicentral.

To help the reader in understanding the term equivalence of SRCAs and right-handed SBAs, it is perhaps useful to provide an explicit axiomatisation of SRCAs. Such an axiomatisation is not long:

1. $q(0, x, y)=y$;

2. $q(w, w, 0)=w$; 
3. $q(w, y, y)=y$;

4. $q(w, q(w, x, y), z)=q(w, x, z)$;

5. $q(w, x, q(w, y, z))=q(w, x, z)$;

6. $q\left(w, q\left(y_{1}, y_{2}, y_{3}\right), q\left(z_{1}, z_{2}, z_{3}\right)\right)=q\left(q\left(w, y_{1}, z_{1}\right), q\left(w, y_{2}, z_{2}\right), q\left(w, y_{3}, z_{3}\right)\right)$.

The last five identities equationally formalise that the element $w$ is semicentral.

Theorem 2.14 ([9]). The variety of right-handed SBAs is term equivalent to the variety of SRCAs.

The proof is based on the following correspondence between the algebraic similarity types of SBAs and of SRCAs:

$$
\begin{aligned}
q(x, y, z) & \rightsquigarrow(x \wedge y) \vee(z \backslash x) \\
x \vee y & \rightsquigarrow q(x, x, y) \\
x \wedge y & \rightsquigarrow q(x, y, 0) \\
x \backslash y & \rightsquigarrow q(y, 0, x) .
\end{aligned}
$$

The natural partial order and preorder of a SRCA are the partial order $\leq$ and the preorder $\preceq_{\mathcal{D}}=\preceq_{\mathcal{R}}$ of its corresponding SBA.

Example 2.15 (see [8,9]). Let $\mathcal{F}(X, Y)$ be the set of all partial functions from $X$ into $Y$. The algebra $\mathbf{F}=(\mathcal{F}(X, Y), q, 0)$ is a SRCA, where

- $0=\emptyset$ is the empty function;

- For all functions $f: F \rightarrow Y, g: G \rightarrow Y$ and $h: H \rightarrow Y(F, G, H \subseteq X)$,

$$
q(f, g, h)=\left.\left.g\right|_{G \cap F} \cup h\right|_{H \cap \bar{F}} .
$$

By Theorem 2.14 $\mathbf{F}$ is term equivalent to the right-handed SBA with universe $\mathcal{F}(X, Y)$, whose operations are defined as follows:

$$
f \wedge g=\left.g\right|_{G \cap F} ; \quad f \vee g=\left.f \cup g\right|_{G \cap \bar{F}} ; \quad g \backslash f=\left.g\right|_{G \cap \bar{F}} .
$$

\section{Boolean-like algebras of finite dimension}

Some important properties of Boolean algebras are shared by $n$-pointed algebras whose elements satisfy all the equational conditions of $n$-central elements through an operator $q$ of arity $n+1$ satisfying the fundamental properties of a generalised if-then-else connective. These algebras, and the varieties they form, were termed Boolean-like algebras of dimension $n$ in [6].

\subsection{Church algebras of finite dimension}

In this section we recall from [6] the notion of a Church algebra of dimension $n$. These algebras have $n$ nullary operations $\mathrm{e}_{1}, \ldots, \mathrm{e}_{n}(n \geq 2)$ and an operation $q$ of arity $n+1$ (a sort of "generalised if-then-else") satisfying the identities $q\left(\mathrm{e}_{i}, x_{1}, \ldots, x_{n}\right)=x_{i}$. The operator $q$ induces, through the so-called $n$-central elements, a decomposition of the algebra into $n$ factors. 
Definition 3.1. Algebras of type $\tau$, equipped with at least $n$ nullary operations $\mathrm{e}_{1}, \ldots, \mathrm{e}_{n}$ $(n \geq 2)$ and a term operation $q$ of arity $n+1$ satisfying $q\left(\mathrm{e}_{i}, x_{1}, \ldots, x_{n}\right)=x_{i}$, are called Church algebras of dimension $n$ ( $n \mathrm{CA}$, for short); $n$ CAs admitting only the $(n+1)$-ary $q$ operator and the $n$ constants $\mathrm{e}_{1}, \ldots, \mathrm{e}_{n}$ are called pure $n$ CAs.

If $\mathbf{A}$ is an $n \mathbf{C A}$, then $\mathbf{A}_{0}=\left(A, q, \mathrm{e}_{1}, \ldots, \mathrm{e}_{n}\right)$ is the pure reduct of $\mathbf{A}$.

Church algebras of dimension 2 were introduced as Church algebras in [20] and studied in [23]. Examples of Church algebras of dimension 2 are Boolean algebras (with $q(x, y, z)=(x \wedge y) \vee(\neg x \wedge z)$ ) or rings with unit (with $q(x, y, z)=x y+z-x z)$. Next, we present some examples of Church algebra having dimension greater than 2 .

Example 3.2 (Semimodules). Let $R$ be a semiring and $V$ be an $R$-semimodule generated by a finite set $E=\left\{\mathrm{e}_{1}, \ldots, \mathrm{e}_{n}\right\}$. Then we define an operation $q$ of arity $n+1$ as follows (for all $\mathbf{v}=\sum_{j=1}^{n} v_{j} \mathrm{e}_{j}$ and $\mathbf{w}^{i}=\sum_{j=1}^{n} w_{j}^{i} \mathrm{e}_{j}$ ):

$$
q\left(\mathbf{v}, \mathbf{w}^{1}, \ldots, \mathbf{w}^{n}\right)=\sum_{i=1}^{n} v_{i} \mathbf{w}^{i} .
$$

Under this definition, $V$ becomes a $n \mathrm{CA}$. As a concrete example, if $B$ is a Boolean algebra, $B^{n}$ is a semimodule (over the Boolean ring $B$ ) with the following operations: $\left(a_{1}, \ldots, a_{n}\right)+\left(b_{1}, \ldots, b_{n}\right)=\left(a_{1} \vee b_{1}, \ldots, a_{n} \vee b_{n}\right)$ and $b\left(a_{1}, \ldots, a_{n}\right)=\left(b \wedge a_{1}, \ldots\right.$, $\left.b \wedge a_{n}\right) . B^{n}$ is also called a Boolean vector space (see $\left.[11,12]\right)$.

Example 3.3 ( $n$-Sets). Let $I$ be a set. A $n$-subset of $I$ is a sequence $\left(Y_{1}, \ldots, Y_{n}\right)$ of subsets $Y_{i}$ of $I$. We denote by $\operatorname{Set}_{n}(I)$ the family of all $n$-subsets of $I$. $\operatorname{Set}_{n}(I)$ becomes a pure $n$ CA if we define an $(n+1)$-ary operator $q$ and $n$ constants $\mathrm{e}_{1}, \ldots, \mathrm{e}_{n}$ as follows, for all $n$-subsets $\mathbf{y}^{i}=\left(Y_{1}^{i}, \ldots, Y_{n}^{i}\right)$ :

$$
\begin{gathered}
q\left(\mathbf{y}^{0}, \mathbf{y}^{1}, \ldots, \mathbf{y}^{n}\right)=\left(\bigcup_{i=1}^{n} Y_{i}^{0} \cap Y_{1}^{i}, \ldots, \bigcup_{i=1}^{n} Y_{i}^{0} \cap Y_{n}^{i}\right) ; \\
\mathrm{e}_{1}=(I, \emptyset, \ldots, \emptyset), \ldots, \mathrm{e}_{n}=(\emptyset, \ldots, \emptyset, I) .
\end{gathered}
$$

In [27], Vaggione introduced the notion of central element to study algebras whose complementary factor congruences can be replaced by certain elements of their universes. Central elements coincide with central idempotents in rings with unit and with members of the centre in ortholattices.

Theorem 3.4 ([6]). If $\mathbf{A}$ is a $n C A$ of type $\tau$ and $c \in A$, then the following conditions are equivalent:

1. $c$ is a factor element (w.r.t. $q$ ) satisfying the identity $q\left(c, \mathrm{e}_{1}, \ldots, \mathrm{e}_{n}\right)=c$;

2. the sequence of congruences $\left(\theta\left(c, \mathrm{e}_{1}\right), \ldots, \theta\left(c, \mathrm{e}_{n}\right)\right)$ is a n-tuple of complementary factor congruences of $\mathbf{A}$;

3. for all $a_{1}, \ldots, a_{n} \in A, q\left(c, a_{1}, \ldots, a_{n}\right)$ is the unique element such that

$$
a_{i} \theta\left(c, \mathrm{e}_{i}\right) q\left(c, a_{1}, \ldots, a_{n}\right),
$$

for all $1 \leq i \leq n$; 
4. The function $f_{c}$, defined by $f_{c}\left(a_{1}, \ldots, a_{n}\right)=q\left(c, a_{1}, \ldots, a_{n}\right)$ for all $a_{1}, \ldots, a_{n} \in$ $A$, is a n-ary decomposition operator on $\mathbf{A}$ such that $f_{c}\left(\mathrm{e}_{1}, \ldots, \mathrm{e}_{n}\right)=c$.

Definition 3.5. If $\mathbf{A}$ is a $n \mathrm{CA}$, then $c \in A$ is called $n$-central if it satisfies one of the equivalent conditions of Theorem 3.4. A $n$-central element $c$ is nontrivial if $c \notin\left\{\mathrm{e}_{1}, \ldots, \mathrm{e}_{n}\right\}$.

Every $n$-central element $c \in A$ induces a decomposition of $\mathbf{A}$ as a direct product of the algebras $\mathbf{A} / \theta\left(c, \mathrm{e}_{i}\right)$, for $i \leq n$.

The set of all $n$-central elements of a $n \mathrm{CA} \mathbf{A}$ is a subalgebra of the pure reduct of $\mathbf{A}$. We denote by $\mathbf{C e}_{n}(\mathbf{A})$ the algebra $\left(\mathrm{Ce}_{n}(\mathbf{A}), q, \mathrm{e}_{1}, \ldots, \mathrm{e}_{n}\right)$ of all $n$-central elements of an $n$ CA A.

Factorisations of arbitrary algebras in $n$ factors may be studied in terms of $n$-central elements of suitable $n$ CAs of functions, as explained in the following example.

Example 3.6. Let $\mathbf{A}$ be an arbitrary algebra of type $\tau$ and $F$ be a set of functions from $A^{n}$ into $A$, which includes the projections $\mathrm{e}_{i}^{\mathbf{F}}$ and all constant functions $f_{b}(b \in A)$ :

(1) $\mathrm{e}_{i}^{\mathbf{F}}\left(a_{1}, \ldots, a_{n}\right)=a_{i}$, for every $a_{1}, \ldots, a_{n} \in A$;

(2) $f_{b}\left(a_{1}, \ldots, a_{n}\right)=b$, for every $a_{1}, \ldots, a_{n} \in A$;

and it is closed under the following operations (for all $f, h_{i}, g_{j} \in F$ and all $a_{1}, \ldots, a_{n} \in$ $A)$ :

(3) $q^{\mathbf{F}}\left(f, g_{1} \ldots, g_{n}\right)\left(a_{1}, \ldots, a_{n}\right)=f\left(g_{1}\left(a_{1}, \ldots, a_{n}\right) \ldots, g_{n}\left(a_{1}, \ldots, a_{n}\right)\right)$.

(4) $\sigma^{\mathbf{F}}\left(h_{1}, \ldots, h_{k}\right)\left(a_{1}, \ldots, a_{n}\right)=\sigma^{\mathbf{A}}\left(h_{1}\left(a_{1}, \ldots, a_{n}\right), \ldots, h_{k}\left(a_{1}, \ldots, a_{n}\right)\right)$, for every $\sigma \in \tau$ of arity $k$.

The algebra $\mathbf{F}=\left(F, \sigma^{\mathbf{F}}, q^{\mathbf{F}}, \mathrm{e}_{1}^{\mathbf{F}}, \ldots, \mathrm{e}_{n}^{\mathbf{F}}\right)_{\sigma \in \tau}$ is a $n$ CA. It is possible to prove that a function $f \in F$ is a $n$-central element of $\mathbf{F}$ if and only if $f$ is a $n$-ary decomposition operator on the algebra A commuting (see Section 2.2) with every function $g \in F$. The reader may consult [22] for the case $n=2$.

\subsection{Boolean-like algebras}

Boolean algebras are Church algebras of dimension 2 all of whose elements are 2-central. It turns out that, among the $n$-dimensional Church algebras, those algebras all of whose elements are $n$-central inherit many of the remarkable properties that distinguish Boolean algebras. We now recall from [6] the notion of Boolean-like algebras of dimension $n$, the main subject of study of this paper.

In [6] $n$ BAs are studied in the general case of an arbitrary similarity type. Here, we restrict ourselves to consider the pure case, where $q$ is the unique operator of the algebra.

Definition 3.7. A pure $n \mathrm{CA} \mathbf{A}=\left(A, q, \mathrm{e}_{1}, \ldots, \mathrm{e}_{n}\right)$ is called a Boolean-like algebra of dimension $n$ ( $n \mathrm{BA}$, for short) if every element of $A$ is $n$-central.

The class of all $n$ BAs is a variety axiomatised by the following identities:

(B0) $q\left(\mathrm{e}_{i}, x_{1}, \ldots, x_{n}\right)=x_{i}(i=1, \ldots, n)$.

(B1) $q(y, x, \ldots, x)=x$. 
(B2)

$$
\begin{aligned}
& q\left(y, q\left(y, x_{11}, x_{12}, \ldots, x_{1 n}\right), \ldots, q\left(y, x_{n 1}, x_{n 2}, \ldots, x_{n n}\right)\right)=q\left(y, x_{11}, \ldots, x_{n n}\right) . \\
& \begin{aligned}
q\left(y, q\left(x_{10}, \ldots, x_{1 n}\right), \ldots, q\left(x_{n 0}, \ldots, x_{n n}\right)\right) \\
=
\end{aligned} \quad q\left(q\left(y, x_{10}, \ldots, x_{n 0}\right), \ldots, q\left(y, x_{1 n}, \ldots, x_{n n}\right)\right) .
\end{aligned}
$$

(B4) $q\left(y, \mathrm{e}_{1}, \ldots, \mathrm{e}_{n}\right)=y$.

In the following lemma we show that every nontrivial $n \mathrm{BA}$ has at least $n$ elements.

Lemma 3.8. The constants $\mathrm{e}_{i}(1 \leq i \leq n)$ are pairwise residually distinct in every nontrivial $n B A$.

Proof. Let $\mathbf{A}$ be a nontrivial $n \mathrm{BA}$ such that $\mathrm{e}_{k}=\mathrm{e}_{j}$ for some $k \neq j$. If $a_{1}, \ldots, a_{n} \in$ $A$ with $a_{k} \neq a_{j}$, then $a_{k}=q\left(\mathrm{e}_{k}, a_{1}, \ldots, a_{n}\right)=q\left(\mathrm{e}_{j}, a_{1}, \ldots, a_{n}\right)=a_{j}$, providing a contradiction.

Boolean-like algebras of dimension 2 were introduced in [23] with the name "Booleanlike algebras". Inter alia, it was shown in that paper that the variety of Boolean-like algebras of dimension 2 is term-equivalent to the variety of Boolean algebras.

Example 3.9. The algebra $\mathbf{C e}_{n}(\mathbf{A})$ of all $n$-central elements of a $n \mathrm{CA} \mathbf{A}$ of type $\tau$ is a canonical example of $n \mathrm{BA}$ (see the remark after Definition 3.5).

Example 3.10. The algebra $\mathbf{n}=\left(\left\{\mathrm{e}_{1}, \ldots, \mathrm{e}_{n}\right\}, q^{\mathbf{n}}, \mathrm{e}_{1}^{\mathbf{n}}, \ldots, \mathrm{e}_{n}^{\mathbf{n}}\right)$, where $q^{\mathbf{n}}\left(\mathrm{e}_{i}, x_{1}, \ldots\right.$, $\left.x_{n}\right)=x_{i}$ for every $i \leq n$, is a $n$ BA.

Example 3.11 (n-Partitions). Let $I$ be a set. An $n$-partition of $I$ is a $n$-subset $\left(Y^{1}, \ldots, Y^{n}\right)$ of $I$ such that $\bigcup_{i=1}^{n} Y^{i}=I$ and $Y^{i} \cap Y^{j}=\emptyset$ for all $i \neq j$. The set of $n$-partitions of $I$ is closed under the $q$-operator defined in Example 3.3 and constitutes the algebra of all $n$-central elements of the pure $n \mathrm{CA} \operatorname{Set}_{n}(I)$ of all $n$-subsets of $I$. Notice that the algebra of $n$-partitions of $I$, denoted by $\operatorname{Par}_{n}(I)$, can be proved isomorphic to the $n \mathrm{BA} \mathbf{n}^{I}$ (the Cartesian product of $I$ copies of the algebra $\mathbf{n}$ ).

The variety $\mathrm{BA}$ of Boolean algebras is semisimple as every $\mathbf{A} \in \mathrm{BA}$ is subdirectly embeddable into a power of the 2-element Boolean algebra, which is the only subdirectly irreducible (in fact, simple) member of BA. This property finds an analogue in the structure theory of $n$ BAs.

Theorem 3.12 ([6]). The algebra $\mathbf{n}$ is the unique subdirectly irreducible (in fact, simple) $n B A$ and it generates the variety of $n B A s$.

The next corollary shows that, for any $n \geq 2$, the $n \mathrm{BA} \mathbf{n}$ plays a role analogous to the Boolean algebra 2 of truth values.

Corollary 3.13. Every nBA A is isomorphic to a subdirect power of $\mathbf{n}^{I}$, for some set $I$.

A subalgebra of the $n \mathrm{BA} \operatorname{Par}_{n}(I)$ of the $n$-partitions on a set $I$, defined in Example 3.11, is called a field of n-partitions on $I$. The Stone representation theorem for $n$ BAs follows.

Corollary 3.14. Any nBA is isomorphic to a field of n-partitions on a suitable set I. 
One of the most remarkable properties of the 2-element Boolean algebra, called primality in universal algebra [7, Section 7 in Chapter IV], is the definability of all finite Boolean functions in terms of the connectives AND, OR, NOT. This property is inherited by $n$ BAs. An algebra of cardinality $n$ is primal if and only if it admits the $n$ BA $\mathbf{n}$ as a subreduct.

Definition 3.15. Let $\mathbf{A}$ be a nontrivial algebra. $\mathbf{A}$ is primal if it is of finite cardinality and, for every function $f: A^{k} \rightarrow A(k \geq 0)$, there is a $k$-ary term $t$ such that for all $a_{1}, \ldots, a_{k} \in A, f\left(a_{1}, \ldots, a_{k}\right)=t^{\mathbf{A}}\left(a_{1}, \ldots, a_{k}\right)$.

A variety $\mathcal{V}$ is primal if $\mathcal{V}=\mathcal{V}(\mathbf{A})$ for a primal algebra $\mathbf{A}$.

\section{Theorem 3.16 ([6]).}

(i) The variety $n \mathrm{BA}=\mathcal{V}(\mathbf{n})$ is primal;

(ii) Let $\mathbf{A}$ be a finite algebra of cardinality $n$. Then $\mathbf{A}$ is primal if and only if it admits the algebra $\mathbf{n}$ as a subreduct.

We would like to point out here that when an algebra $\mathbf{A}$ is primal, the choice of fundamental operations is a matter of taste and convenience (since any set of functionally complete operations would serve), and hence is typically driven by applications.

\section{Skew Boolean algebras and $n$ BAs}

In this section we prove that any $n \mathrm{BA} \mathbf{A}$ contains a symmetric $\cap$-skew cluster of righthanded SBIAs $S_{1}^{\cap}(\mathbf{A}), \ldots, S_{n}^{\cap}(\mathbf{A})$. The algebra $S_{i}^{\cap}(\mathbf{A})$, called the $\cap$-skew $i$-reduct of $\mathbf{A}$, has $\mathrm{e}_{i}$ as a bottom element, and the other constants $\mathrm{e}_{1}, \ldots, \mathrm{e}_{i-1}, \mathrm{e}_{i+1}, \ldots, \mathrm{e}_{n}$ as maximal elements. Rather interestingly, every permutation $\sigma$ of the symmetric group $S_{n}$ determines a bunch of isomorphisms

$$
\mathrm{S}_{1}^{\cap}(\mathbf{A}) \cong \mathrm{S}_{\sigma 1}^{\cap}(\mathbf{A}), \ldots, \mathrm{S}_{n}^{\cap}(\mathbf{A}) \cong \mathrm{S}_{\sigma n}^{\cap}(\mathbf{A})
$$

which shows the inner symmetry of the $n$ BAs. Every $n$ BA has also a skew cluster $S_{1}(\mathbf{A})$, $\ldots, S_{n}(\mathbf{A})$ of isomorphic right-handed SBAs, which are the skew Boolean algebra reducts of members of the $\cap$-skew cluster of $\mathbf{A}$. We conclude the section with a general representation theorem for right-handed SBAs in terms of $n$ BAs of $n$-partitions.

\subsection{The skew reducts of a $n \mathrm{BA}$}

In [9] it is shown that the variety of SBAs is term equivalent to the variety of SRCAs (see Section 2.6), whose type contains only a ternary operator and a nullary operator. Here we use the $(n+1)$-ary operator $q$ of a $n$ BA A to define ternary operators $t_{1}, \ldots, t_{n}$ such that the reducts $\left(A, t_{i}, \mathrm{e}_{i}\right)$ are isomorphic SRCAs. Their term equivalent SBAs are all isomorphic reducts of $\mathbf{A}$, too. We also show that these isomorphic SBAs are in their turn reducts of isomorphic SBIAs.

For every $i \in \hat{n}$, we denote by $\bar{i}$ the set $\hat{n} \backslash\{i\}$.

In the following definition we use the $(n+1)$-ary operator $q$ of $n$ BAs to introduce some term operations needed to define the above-described reducts of $n \mathrm{BAs}$.

Definition 4.1. Let $\mathbf{A}=\left(A, q, \mathrm{e}_{1}, \ldots, \mathrm{e}_{n}\right)$ be a $n \mathrm{BA}$. Given $1 \leq i \leq n$, we define the following term operations: 
- $t_{i}(x, y, z)=q(x, y / \bar{i}, z / i)$;

- $x \wedge_{i} y=t_{i}\left(x, y, \mathrm{e}_{i}\right)$;

- $x \vee_{i} y=t_{i}(x, x, y)$;

- $x \backslash_{i} y=t_{i}\left(y, \mathrm{e}_{i}, x\right)$;

- $d_{i}(x, y)=q\left(x, t_{1}\left(y, x \vee_{i} y, \mathrm{e}_{i}\right), t_{2}\left(y, x \vee_{i} y, \mathrm{e}_{i}\right), \ldots, t_{n}\left(y, x \vee_{i} y, \mathrm{e}_{i}\right)\right)$;

- $x \cap_{i} y=q\left(x, t_{1}\left(y, \mathrm{e}_{i}, x\right), t_{2}\left(y, \mathrm{e}_{i}, x\right), \ldots, t_{n}\left(y, \mathrm{e}_{i}, x\right)\right)$.

We now define three reducts of a $n \mathrm{BA} \mathbf{A}$, for each $1 \leq i \leq n$.

Definition 4.2. Let $\mathbf{A}$ be a $n \mathrm{BA}$. We define the following three reducts of $\mathbf{A}$ :

(i) The right Church $i$-reduct $R_{i}(\mathbf{A})=\left(A, t_{i}, \mathrm{e}_{i}\right)$.

(ii) The skew $i$-reduct $S_{i}(\mathbf{A})=\left(A, \wedge_{i}, \vee_{i}, \backslash_{i}, \mathrm{e}_{i}\right)$.

(iii) The $\cap$-skew $i$-reduct $S_{i}^{\cap}(\mathbf{A})=\left(A, \wedge_{i}, \vee_{i}, \backslash_{i}, \mathrm{e}_{i}, \cap_{i}\right)$.

In the remaining part of this subsection we will prove that $R_{i}(\mathbf{A})$ is a SRCA, $S_{i}^{\cap}(\mathbf{A})$ is a $e_{i}$-regular (w.r.t. $d_{i}$ ) right-handed SBIA, and $S_{i}(\mathbf{A})$ is a right-handed SBA.

In the following lemmas we prove some properties of the term operations introduced in Definition 4.1.

Lemma 4.3. The term operations of Definition 4.1 satisfy the following conditions when they are interpreted in the generator $\mathbf{n}$ of the variety $n B A$ :

$$
\begin{aligned}
& t_{i}(a, b, c)=\left\{\begin{array}{ll}
c & \text { if } a=\mathrm{e}_{i} \\
b & \text { if } a \neq \mathrm{e}_{i}
\end{array} ; \quad a \wedge_{i} b=\left\{\begin{array}{ll}
\mathrm{e}_{i} & \text { if } a=\mathrm{e}_{i} \\
b & \text { if } a \neq \mathrm{e}_{i}
\end{array} ; \quad a \vee_{i} b=\left\{\begin{array}{ll}
b & \text { if } a=\mathrm{e}_{i} \\
a & \text { if } a \neq \mathrm{e}_{i}
\end{array} ;\right.\right.\right. \\
& a \backslash_{i} b=\left\{\begin{array}{ll}
a & \text { if } b=\mathrm{e}_{i} \\
\mathrm{e}_{i} & \text { if } b \neq \mathrm{e}_{i}
\end{array} ; \quad d_{i}(a, b)=\left\{\begin{array}{ll}
\mathrm{e}_{i} & \text { if } a=b \\
a \vee_{i} b & \text { if } a \neq b
\end{array} ; \quad a \cap_{i} b=\left\{\begin{array}{ll}
a & \text { if } a=b \\
\mathrm{e}_{i} & \text { if } a \neq b
\end{array} .\right.\right.\right.
\end{aligned}
$$

Proof. The proof is trivial for $t_{i}, \wedge_{i}, \vee_{i}, \backslash_{i}$. We now prove the relation for $d_{i}(a, b)$. We distinguish three cases.

- $(a=b)$ :

$$
\begin{aligned}
d_{i}(a, a) & =q\left(a, t_{1}\left(a, a \vee_{i} a, \mathrm{e}_{i}\right), \ldots, t_{n}\left(a, a \vee_{i} a, \mathrm{e}_{i}\right)\right) \\
& ={ }_{(\mathrm{B} 2)} q\left(a, \mathrm{e}_{i}, \ldots, \mathrm{e}_{i}\right)={ }_{(\mathrm{B} 1)} \mathrm{e}_{i} .
\end{aligned}
$$

- $\left(a=\mathrm{e}_{k}\right.$ and $\left.a \neq b\right)$ :

$$
\begin{aligned}
d_{i}\left(\mathrm{e}_{k}, b\right) & =q\left(\mathrm{e}_{k}, t_{1}\left(b, \mathrm{e}_{k} \vee_{i} b, \mathrm{e}_{i}\right), \ldots, t_{n}\left(b, \mathrm{e}_{k} \vee_{i} b, \mathrm{e}_{i}\right)\right) \\
& =t_{k}\left(b, \mathrm{e}_{k} \vee_{i} b, \mathrm{e}_{i}\right)={ }_{\left(b \neq \mathrm{e}_{k}\right)} \mathrm{e}_{k} \vee_{i} b=a \vee_{i} b
\end{aligned}
$$

By definition of $\cap_{i}$ it is trivial to prove $a \cap_{i} a=a$. If $a=\mathrm{e}_{k} \neq b$, then we have:

$$
\begin{aligned}
a \cap_{i} b & =q\left(a, t_{1}\left(b, \mathrm{e}_{i}, a\right), t_{2}\left(b, \mathrm{e}_{i}, a\right), \ldots, t_{n}\left(b, \mathrm{e}_{i}, a\right)\right) \\
& ={ }_{\left(a=\mathrm{e}_{k}\right)} t_{k}\left(b, \mathrm{e}_{i}, a\right)={ }_{\left(b \neq \mathrm{e}_{k}\right)} \mathrm{e}_{i} .
\end{aligned}
$$


Lemma 4.4. The following identities hold in every $n B A$ :

(1) $t_{i}\left(\mathrm{e}_{i}, x, y\right)=y$ and $t_{i}\left(\mathrm{e}_{j}, x, y\right)=x$, for every $j \neq i$;

(2)

$$
\begin{aligned}
q\left(x, y_{1}, \ldots, y_{n}\right) & =t_{1}\left(x, t_{2}\left(x, t_{3}\left(x, \ldots t_{n}\left(x, z, y_{n}\right) \ldots, y_{3}\right), y_{2}\right), y_{1}\right) \\
& =t_{1}\left(x, t_{2}\left(x, t_{3}\left(x, \ldots t_{n-1}\left(x, y_{n}, y_{n-1}\right) \ldots, y_{3}\right), y_{2}\right), y_{1}\right) .
\end{aligned}
$$

(3) $\wedge_{i}$ and $\vee_{i}$ are idempotents;

(4) $d_{i}(x, x)=\mathrm{e}_{i}$ and $x \cap_{i} x=x$;

(5) $t_{i}\left(d_{i}(x, y), x, y\right)=x$ and $t_{i}\left(x \cap_{i} y, y, x\right)=x$;

(6) $\left(A, \cap_{i}, \mathrm{e}_{i}\right)$ is a meet semilattice with bottom $\mathrm{e}_{i}$;

(7) $x \cap_{i}\left(x \wedge_{i} y \wedge_{i} x\right)=x \wedge_{i} y \wedge_{i} x$ and $x \wedge_{i}\left(x \cap_{i} y\right)=x \cap_{i} y=\left(x \cap_{i} y\right) \wedge_{i} x$;

(8) $d_{i}(x, y)=\left(x \vee_{i} y\right) \backslash_{i}\left(x \cap_{i} y\right)$;

(9) $x \cap_{i} y=\left(x \wedge_{i} y\right) \backslash_{i} d_{i}(x, y)$.

Proof. The identities are checked in the generator $\mathbf{n}$ of the variety $n \mathrm{BA}$.

(2): First we have:

$$
\begin{aligned}
t_{1}\left(\mathrm{e}_{k}, t_{2}\left(\mathrm{e}_{k}, t_{3}\right.\right. & \left.\left.\left(\mathrm{e}_{k}, \ldots t_{n}\left(\mathrm{e}_{k}, c, b_{n}\right) \ldots, b_{3}\right), b_{2}\right), b_{1}\right) \\
& =t_{2}\left(\mathrm{e}_{k}, t_{3}\left(\mathrm{e}_{k}, \ldots t_{n}\left(\mathrm{e}_{k}, c, b_{n}\right) \ldots, b_{3}\right), b_{2}\right) \\
& =\cdots=t_{k}\left(\mathrm{e}_{k}, \ldots t_{n}\left(\mathrm{e}_{k}, c, b_{n}\right) \ldots, b_{k}\right)=b_{k}=q\left(\mathrm{e}_{k}, b_{1}, \ldots, b_{n}\right) .
\end{aligned}
$$

If $k \neq n$, a similar computation gives:

$$
t_{1}\left(\mathrm{e}_{k}, t_{2}\left(\mathrm{e}_{k}, t_{3}\left(\mathrm{e}_{k}, \ldots t_{n-1}\left(\mathrm{e}_{k}, b_{n}, b_{n-1}\right) \ldots, b_{3}\right), b_{2}\right), b_{1}\right)=b_{k} .
$$

If $k=n$, then we have:

$$
\begin{array}{r}
t_{1}\left(\mathrm{e}_{n}, t_{2}\left(\mathrm{e}_{n}, t_{3}\left(\mathrm{e}_{n}, \ldots t_{n-1}\left(\mathrm{e}_{n}, b_{n}, b_{n-1}\right)\right.\right.\right. \\
\left.\left.\left.\quad \ldots, b_{3}\right), b_{2}\right), b_{1}\right) \\
=\cdots=t_{n-1}\left(\mathrm{e}_{n}, b_{n}, b_{n-1}\right)=b_{n} .
\end{array}
$$

(3) - (4): Trivial by Lemma 4.3.

(5): If $a=b$, then the conclusion $t_{i}\left(d_{i}(a, a), a, a\right)=a$ and $t_{i}\left(a \cap_{i} a, a, a\right)=a$ is trivial by (4). Let now $a \neq b$.

- $t_{i}\left(a \cap_{i} b, b, a\right)={ }_{(\operatorname{Lemma~4.3)}} t_{i}\left(\mathrm{e}_{i}, b, a\right)=a$.

- If $a=\mathrm{e}_{i}$ then $t_{i}\left(d_{i}\left(\mathrm{e}_{i}, b\right), \mathrm{e}_{i}, b\right)={ }_{(\operatorname{Lemma~4.3)}} t_{i}\left(b, \mathrm{e}_{i}, b\right)={ }_{\left(b \neq \mathrm{e}_{i}\right)} \mathrm{e}_{i}=a$.

- If $a \neq \mathrm{e}_{i}$, then $t_{i}\left(d_{i}(a, b), a, b\right)==_{(\operatorname{Lemma} 4.3)} t_{i}(a, a, b)={ }_{\left(a \neq \mathrm{e}_{i}\right)} a$.

(8):

$$
\begin{aligned}
\left(a \vee_{i} b\right) \backslash_{i}\left(a \cap_{i} b\right) & =t_{i}\left(a \cap_{i} b, \mathrm{e}_{i}, a \vee_{i} b\right) \\
& = \begin{cases}t_{i}\left(a, \mathrm{e}_{i}, a\right)=\mathrm{e}_{i} & \text { if } a=b \\
t_{i}\left(\mathrm{e}_{i}, \mathrm{e}_{i}, a \vee_{i} b\right)=a \vee_{i} b & \text { if } a \neq b\end{cases} \\
& =d_{i}(a, b) .
\end{aligned}
$$


(9): First we have: $\left(a \wedge_{i} a\right) \backslash_{i} d_{i}(a, a)=t_{i}\left(\mathrm{e}_{i}, \mathrm{e}_{i}, a\right)=a=a \cap_{i} a$. If $a \neq b$, then

$$
\begin{aligned}
\left(a \wedge_{i} b\right) \backslash_{i} d_{i}(a, b) & =t_{i}\left(d_{i}(a, b), \mathrm{e}_{i}, a \wedge_{i} b\right) \\
& =t_{i}\left(a \vee_{i} b, \mathrm{e}_{i}, a \wedge_{i} b\right)=\mathrm{e}_{i}=a \cap_{i} b
\end{aligned}
$$

because by Lemma 4.3, $a \vee_{i} b \neq \mathrm{e}_{i}$ if $a \neq b$.

(6) and (7) can be similarly checked in the generator $\mathbf{n}$ of the variety $n$ BA by using Lemma 4.3.

Lemma 4.5. Let $\mathbf{A}$ be a $n B A$, and $a, b \in A$. Then we have:

(i) $t_{i}(a,-,-)$ is a 2-ary decomposition operator on $\mathbf{A}$.

(ii) $d_{i}(a, b)=\mathrm{e}_{i} \Rightarrow a=b$.

Proof. (i): The binary operator $t_{i}(a,-,-)$ is a decomposition operator, because it is obtained by the $n$-ary decomposition operator $q(a,-, \ldots,-)$ equating some of its coordinates (see [21] and Proposition 2.5).

(ii): Let $d_{i}(a, b)=\mathrm{e}_{i}$. Then $a==_{(\operatorname{Lemma} 4.4(5))} t_{i}\left(d_{i}(a, b), a, b\right)=t_{i}\left(\mathrm{e}_{i}, a, b\right)=b$.

We now characterise the reducts $R_{i}(\mathbf{A}), S_{i}(\mathbf{A})$ and $S_{i}^{\bigcap}(\mathbf{A})$ of a $n$ BA A (see Definition 4.2).

Proposition 4.6. Let $\mathbf{A}$ be a $n B A$. Then the following conditions hold:

(i) The right Church $i$-reduct $R_{i}(\mathbf{A})=\left(A, t_{i}, \mathrm{e}_{i}\right)$ of $\mathbf{A}$ is a SRCA;

(ii) The skew $i$-reduct $S_{i}(\mathbf{A})=\left(A, \wedge_{i}, \vee_{i}, \backslash_{i}, \mathrm{e}_{i}\right)$ of $\mathbf{A}$ is a right-handed $S B A$.

Proof. By Lemma 4.4(3) and Lemma 4.5(i) every element of $A$ is a factor element (w.r.t. $t_{i}$ ) that is $\wedge_{i}$-idempotent. Then every element of $A$ is semicentral, so that $R_{i}(\mathbf{A})$ is a SRCA. By Theorem 2.14 the skew $i$-reduct $S_{i}(\mathbf{A})$ is a right-handed SBA.

Hereafter, we denote by $\preceq_{\mathcal{D}}^{i}, \preceq_{\mathcal{L}}^{i}, \preceq_{\mathcal{R}}^{i}$ and $\leq^{i}$ the natural preorders and order of the SBA $S_{i}(\mathbf{A})$ (see Section 2.4). Since $S_{i}(\mathbf{A})$ is right-handed $\preceq_{\mathcal{D}}^{i}$ and $\preceq_{\mathcal{R}}^{i}$ coincide.

Proposition 4.7. The elements $\mathrm{e}_{1}, \ldots, \mathrm{e}_{i-1}, \mathrm{e}_{i+1}, \ldots, \mathrm{e}_{n}$ are maximal elements of $S_{i}(\mathbf{A})$.

Proof. We show the maximality of the elements $\mathrm{e}_{1}, \ldots, \mathrm{e}_{i-1}, \mathrm{e}_{i+1}, \ldots, \mathrm{e}_{n}$ with respect to the natural preorder $\preceq_{\mathcal{D}}^{i}$ of the SBA $S_{i}(\mathbf{A})$, defined by $a \preceq_{\mathcal{D}}^{i} b$ iff $a \wedge_{i} b \wedge_{i} a=a$. If $k \neq i$ and $a \in A$, then $a \wedge_{i} \mathrm{e}_{k} \wedge_{i} a=a \wedge_{i} a=a$, because $\mathrm{e}_{k} \wedge_{i} a=t_{i}\left(\mathrm{e}_{k}, a, \mathrm{e}_{i}\right)=a$ by Lemma 4.4(1).

By Proposition 4.7 the skew $i$-reduct $S_{i}(\mathbf{A})$ has a maximal class $M$ with $\mathrm{e}_{1}, \ldots, \mathrm{e}_{i-1}$, $\mathrm{e}_{i+1}, \ldots, \mathrm{e}_{n} \in M$. Then the algebra $S_{i}(\mathbf{A}) / \mathcal{D}_{i}$ is a Boolean algebra, where $\mathcal{D}_{i}$ is the equivalence induced by $\preceq_{\mathcal{D}}^{i}$.

Proposition 4.8. The $\cap$-skew $i$-reduct $S_{i}^{\cap}(\mathbf{A})=\left(A, \wedge_{i}, \vee_{i}, \backslash_{i}, \mathrm{e}_{i}, \cap_{i}\right)$ of $\mathbf{A}$ is a $\mathrm{e}_{i}$-regular right-handed SBIA.

Proof. By Proposition 4.6 the skew $i$-reduct $S_{i}(\mathbf{A})=\left(A, \wedge_{i}, \vee_{i}, \backslash_{i}, \mathrm{e}_{i}\right)$ is a right-handed SBA. By Lemma 4.4(6),(7) and by Definition 2.9 the $\cap$-skew $i$-reduct $S_{i}^{\cap}(\mathbf{A})$ is a righthanded SBIA. By Lemma 4.4(8) we have that $d_{i}(x, y)=\left(x \vee_{i} y\right) \backslash_{i}\left(x \cap_{i} y\right)$ is a term operation in the type of SBIAs. Then the $e_{i}$-regularity w.r.t. $d_{i}$ follows from Lemma 4.4(4) and Lemma 4.5(ii) (see Section 2.1 for the definition of regularity). 
Remark 4.9. Skew Boolean algebras, whose underlying natural partial ordering is a meet semilattice, cannot be equationally axiomatised in the type of SBAs. Therefore, skew Boolean $\cap$-algebras of Definition 2.9 are equationally axiomatised in the type of SBAs enriched with a binary operator $\cap$ of intersection. Rather interestingly, if $\mathbf{A}$ is a $n \mathrm{BA}$ the term operation $\cap_{i}$ is definable in terms of $\wedge_{1}, \vee_{1}, \backslash_{1}, \mathrm{e}_{1}, \ldots, \wedge_{n}, \vee_{n}, \backslash_{n}, \mathrm{e}_{n}$. This follows from Definition 4.1, Lemma 4.4(2), and Theorem 2.14. Then the following question is natural. Let $\mathbf{A}=(A, \wedge, \vee, \backslash, 0)$ be a SBA, whose underlying natural partial ordering is a meet semilattice $(A, \cap, 0)$ with bottom. Does there exist a bunch of SBAs $\mathbf{A}_{1}=\left(A, \wedge_{1}, \vee_{1}, \backslash_{1}, 0_{1}\right), \ldots, \mathbf{A}_{k}=\left(A, \wedge_{k}, \vee_{k}, \backslash_{k}, 0_{k}\right)$ such that the meet operation $\cap$ is definable in terms of the skew Boolean operations of $\mathbf{A}_{1}, \ldots, \mathbf{A}_{k}$ ? A further analysis of this question will be given in Section 5 .

\subsection{A bunch of isomorphisms}

It turns out that all the $\cap$-skew reducts of a $n \mathrm{BA} \mathbf{A}$ are isomorphic. In order to prove this, we study the action of the symmetric group $S_{n}$ on $\mathbf{A}$. The first part of this section is rather technical.

Let $\mathbf{A}$ be a $n$ BA. For every permutation $\sigma$ of the symmetric group $S_{n}$ and $a, b_{1}, \ldots, b_{n} \in$ $A$, we define a sequence $u_{s}(1 \leq s \leq n+1)$ parametrised by another permutation $\tau$ :

$$
u_{n+1}=b_{\tau n} ; \quad u_{s}=t_{\tau s}\left(a, u_{s+1}, b_{\sigma \tau s}\right) \quad(1 \leq s \leq n) .
$$

In the following lemma we prove that $u_{1}$ is independent of the permutation $\tau$.

Notice that $u_{n}=q\left(a, b_{\tau n} / \overline{\tau n}, b_{\sigma \tau n} / \tau n\right)$ and $u_{s}=q\left(a, u_{s+1} / \overline{\tau s}, b_{\sigma \tau s} / \tau s\right)$.

Lemma 4.10. For every $1 \leq s \leq n$ we have:

$$
u_{s}=q\left(a, b_{\tau n} /\{\tau 1, \tau 2 \ldots, \tau(s-1)\}, b_{\sigma \tau s} / \tau s, b_{\sigma \tau(s+1)} / \tau(s+1), \ldots, b_{\sigma \tau n} / \tau n\right) .
$$

Then $u_{1}=q\left(a, b_{\sigma \tau 1} / \tau 1, b_{\sigma \tau 2} / \tau 2, \ldots, b_{\sigma \tau n} / \tau n\right)=q\left(a, b_{\sigma 1}, b_{\sigma 2}, \ldots, b_{\sigma n}\right)$.

Proof. Assume that

$$
u_{s+1}=q\left(a, b_{\tau n} /\{\tau 1, \tau 2 \ldots, \tau s\}, b_{\sigma \tau(s+1)} / \tau(s+1), \ldots, b_{\sigma \tau n} / \tau n\right) .
$$

Then we have:

$$
\begin{aligned}
u_{s} & =t_{\tau s}\left(a, u_{s+1}, b_{\sigma \tau s}\right) \\
& =q\left(a, u_{s+1} / \overline{\tau s}, b_{\sigma \tau s} / \tau s\right) \\
& ={ }_{(\mathrm{B} 2)} q\left(a, b_{\tau n} /\{\tau 1, \tau 2 \ldots, \tau(s-1)\}, b_{\sigma \tau s} / \tau s, b_{\sigma \tau(s+1)} / \tau(s+1), \ldots, b_{\sigma \tau n} / \tau n\right)
\end{aligned}
$$

We define

$$
a^{\sigma}=q\left(a, \mathrm{e}_{\sigma 1}, \mathrm{e}_{\sigma 2}, \ldots, \mathrm{e}_{\sigma n}\right)
$$

The transposition $(i j)$ exchanges $i$ and $j:(i j)(i)=j$ and $(i j)(j)=i$.

Lemma 4.11. The following conditions hold in every $n B A$, for all permutations $\sigma, \tau$ and indices $i \neq j$ :

(1) The 2-ary decomposition operators $t_{i}(x,-,-)$ and $t_{j}(x,-,-)$ commute:

$$
\begin{aligned}
t_{i}\left(x, t_{j}(x, y, z), t_{j}(x, u, w)\right) & =t_{j}\left(x, t_{i}(x, y, u), t_{i}(x, z, w)\right) \\
& =q(x, y / \overline{\{i, j\}}, z / j, u / i)
\end{aligned}
$$


(2) $t_{i}\left(x, t_{j}(x, y, z), u\right)=t_{j}\left(x, t_{i}(x, y, u), z\right)=q(x, y / \overline{\{i, j\}}, z / j, u / i)$;

(3) $q\left(x, y_{\sigma 1}, \ldots, y_{\sigma n}\right)$

$$
\left.=t_{\tau 1}\left(x, t_{\tau 2}\left(x, t_{\tau 3}\left(x, \ldots t_{\tau n}\left(x, y_{\tau n}, y_{\sigma \tau n}\right) \ldots, y_{\sigma \tau 3}\right)\right), y_{\sigma \tau 2}\right), y_{\sigma \tau 1}\right) \text {; }
$$

(4) $\left.x^{\sigma}=t_{\tau 1}\left(x, t_{\tau 2}\left(x, t_{\tau 3}\left(x, \ldots t_{\tau n}\left(x, \mathrm{e}_{\tau n}, \mathrm{e}_{\sigma \tau n}\right) \ldots, \mathrm{e}_{\sigma \tau 3}\right)\right), \mathrm{e}_{\sigma \tau 2}\right), \mathrm{e}_{\sigma \tau 1}\right)$;

(5) $q\left(x^{\sigma}, y_{1}, \ldots, y_{n}\right)=q\left(x, y_{\sigma 1}, \ldots, y_{\sigma n}\right)$;

(6) $x^{(i j)}=t_{i}\left(x, t_{j}\left(x, x, \mathrm{e}_{i}\right), \mathrm{e}_{j}\right)=t_{j}\left(x, t_{i}\left(x, x, \mathrm{e}_{j}\right), \mathrm{e}_{i}\right)$;

(7) $q\left(x, y_{1}, \ldots, y_{n}\right)^{\sigma}=q\left(x,\left(y_{1}\right)^{\sigma}, \ldots,\left(y_{n}\right)^{\sigma}\right)$;

(8) $x^{\tau \circ \sigma}=\left(x^{\sigma}\right)^{\tau}$.

Proof. Let $\mathbf{A}$ be a $n \mathrm{BA}$ and $a, b, c, d, e, b_{1}, \ldots, b_{n}$ be elements of $A$.

(1):

$$
\begin{aligned}
t_{i}\left(a, t_{j}(a, b, c), t_{j}(a, d, e)\right) & =q\left(a, t_{j}(a, b, c) / \bar{i}, t_{j}(a, d, e) / i\right) \\
& =q(a, q(a, b / \bar{j}, c / j) / \bar{i}, q(a, d / \bar{j}, e / j) / i) \\
& ={ }_{(\mathrm{B} 2)} q(a, b / \overline{\{i, j\}}, c / j, d / i) .
\end{aligned}
$$

By symmetry we also get $t_{j}\left(a, t_{i}(a, b, d), t_{i}(a, c, e)\right)=q(a, b / \overline{\{i, j\}}, c / j, d / i)$.

(2): $t_{i}\left(a, t_{j}(a, b, c), d\right)=t_{i}\left(a, t_{j}(a, b, c), t_{j}(a, d, d)\right)={ }_{(1)} q(a, b / \overline{\{i, j\}}, c / j, d / i)$, and similarly $t_{j}\left(a, t_{i}(a, b, d), c\right)=t_{j}\left(a, t_{i}(a, b, d), t_{i}(a, c, c)\right)=q(a, b / \overline{\{i, j\}}, c / j, d / i)$.

(3): By Lemma 4.10, $u_{1}=q\left(a, b_{\sigma 1}, \ldots, b_{\sigma n}\right)$. Then the conclusion follows from the unfolding of the definition of $u_{1}$ :

$$
u_{1}=t_{\tau 1}\left(a, u_{2}, b_{\sigma \tau 1}\right)=t_{\tau 1}\left(a, t_{\tau 2}\left(a, u_{3}, b_{\sigma \tau 2}\right), b_{\sigma \tau 1}\right)=\cdots
$$

(4): Follows from (3) by putting $y_{k}=\mathrm{e}_{k}$.

(5): $q\left(a^{\sigma}, b_{1}, \ldots, b_{n}\right)=q\left(q\left(a, \mathrm{e}_{\sigma 1}, \ldots, \mathrm{e}_{\sigma n}\right), b_{1}, \ldots, b_{n}\right)={ }_{(\mathrm{B} 3)} q\left(a, b_{\sigma 1}, \ldots, b_{\sigma n}\right)$.

(6):

$$
\begin{aligned}
t_{i}\left(a, t_{j}\left(a, a, \mathrm{e}_{i}\right), \mathrm{e}_{j}\right) & =t_{j}\left(a, t_{i}\left(a, a, \mathrm{e}_{j}\right), \mathrm{e}_{i}\right) \\
& =q\left(a, a / \overline{\{i, j\}}, \mathrm{e}_{i} / j, \mathrm{e}_{j} / i\right) \\
& =q\left(a, q\left(a, \mathrm{e}_{1}, \ldots, \mathrm{e}_{n}\right) / \overline{\{i, j\}}, \mathrm{e}_{i} / j, \mathrm{e}_{j} / i\right) \\
& =a^{(i j)}
\end{aligned}
$$

(7):

$$
\begin{aligned}
q\left(a, b_{1}, \ldots, b_{n}\right)^{\sigma} & =q\left(q\left(a, b_{1}, \ldots, b_{n}\right), \mathrm{e}_{\sigma 1}, \mathrm{e}_{\sigma 2}, \ldots, \mathrm{e}_{\sigma n}\right) \\
& ={ }_{(\text {B3 })} q\left(a, q\left(b_{1}, \mathrm{e}_{\sigma 1}, \mathrm{e}_{\sigma 2}, \ldots, \mathrm{e}_{\sigma n}\right), \ldots, q\left(b_{n}, \mathrm{e}_{\sigma 1}, \mathrm{e}_{\sigma 2}, \ldots, \mathrm{e}_{\sigma n}\right)\right) \\
& =q\left(a,\left(b_{1}\right)^{\sigma}, \ldots,\left(b_{n}\right)^{\sigma}\right) .
\end{aligned}
$$

(8):

$$
\begin{aligned}
\left(a^{\sigma}\right)^{\tau} & =q\left(a, \mathrm{e}_{\sigma 1}, \mathrm{e}_{\sigma 2}, \ldots, \mathrm{e}_{\sigma n}\right)^{\tau} \\
& ={ }_{(7)} q\left(a,\left(\mathrm{e}_{\sigma 1}\right)^{\tau}, \ldots,\left(\mathrm{e}_{\sigma n}\right)^{\tau}\right) \\
& =q\left(a, \mathrm{e}_{\tau(\sigma 1)}, \ldots, \mathrm{e}_{\tau(\sigma n)}\right) \\
& =a^{\tau \circ \sigma} .
\end{aligned}
$$


Theorem 4.12. For every transposition $(r k) \in S_{n}$, the map $x \mapsto x^{(r k)}$ defines an isomorphism from $\mathrm{S}_{r}^{\cap}(\mathbf{A})$ onto $\mathrm{S}_{k}^{\cap}(\mathbf{A})$.

Proof. Let $\sigma=(r k)$ in this proof. The map $x \mapsto x^{\sigma}$ is bijective, because

$$
\left(a^{\sigma}\right)^{\sigma}={ }_{(\operatorname{Lemma} 4.11(8))} a^{\sigma \circ \sigma}=a^{\mathrm{Id}}=q\left(a, \mathrm{e}_{1}, \ldots, \mathrm{e}_{n}\right)={ }_{(\mathrm{B} 4)} a,
$$

for every $a \in A$. We now prove that $x \mapsto x^{\sigma}$ is a homomorphism of SBIAs. We recall from Definition 4.1 that the operations $\wedge_{r}, \vee_{r}, \backslash_{r}$ are defined in terms of $t_{r}$. Then to get the conclusion it is sufficient to prove the following equalities, for all $a, b, c \in A: t_{r}(a, b, c)^{\sigma}=$ $t_{k}\left(a^{\sigma}, b^{\sigma}, c^{\sigma}\right),\left(a \cap_{r} b\right)^{\sigma}=a^{\sigma} \cap_{k} b^{\sigma}$ and $\left(\mathrm{e}_{r}\right)^{\sigma}=\mathrm{e}_{k}$ :

$$
\begin{aligned}
& t_{r}(a, b, c)^{\sigma}={ }_{(\operatorname{Lemma} 4.11(7))} t_{r}\left(a, b^{\sigma}, c^{\sigma}\right)=t_{r}\left(\left(a^{\sigma}\right)^{\sigma}, b^{\sigma}, c^{\sigma}\right)=q\left(\left(a^{\sigma}\right)^{\sigma}, b^{\sigma} / \bar{r}, c^{\sigma} / r\right) \\
& ={ }_{(\text {Lemma 4.11(5)) }} q\left(a^{\sigma}, b^{\sigma} / \bar{k}, c^{\sigma} / k\right)=t_{k}\left(a^{\sigma}, b^{\sigma}, c^{\sigma}\right) . \\
& \text { - }\left(\mathrm{e}_{r}\right)^{\sigma}=\mathrm{e}_{\sigma r}=\mathrm{e}_{k} \text {. } \\
& \left(a \cap_{r} b\right)^{\sigma}=q\left(a, t_{1}\left(b, \mathrm{e}_{r}, a\right), \ldots, t_{n}\left(b, \mathrm{e}_{r}, a\right)\right)^{\sigma} \\
& =q\left(a, t_{1}\left(b, \mathrm{e}_{r}, a\right)^{\sigma}, \ldots, t_{n}\left(b, \mathrm{e}_{r}, a\right)^{\sigma}\right) \\
& =q\left(a, t_{1}\left(b,\left(\mathrm{e}_{r}\right)^{\sigma}, a^{\sigma}\right), \ldots, t_{n}\left(b,\left(\mathrm{e}_{r}\right)^{\sigma}, a^{\sigma}\right)\right) \\
& =q\left(a, t_{1}\left(b, \mathrm{e}_{k}, a^{\sigma}\right), \ldots, t_{n}\left(b, \mathrm{e}_{k}, a^{\sigma}\right)\right) \\
& =q\left(\left(a^{\sigma}\right)^{\sigma}, t_{1}\left(\left(b^{\sigma}\right)^{\sigma}, \mathrm{e}_{k}, a^{\sigma}\right), \ldots, t_{n}\left(\left(b^{\sigma}\right)^{\sigma}, \mathrm{e}_{k}, a^{\sigma}\right)\right) \\
& =q\left(\left(a^{\sigma}\right)^{\sigma}, \ldots, t_{r}\left(\left(b^{\sigma}\right)^{\sigma}, \mathrm{e}_{k}, a^{\sigma}\right), \ldots, t_{k}\left(\left(b^{\sigma}\right)^{\sigma}, \mathrm{e}_{k}, a^{\sigma}\right), \ldots\right) \\
& =q\left(a^{\sigma}, \ldots, t_{k}\left(\left(b^{\sigma}\right)^{\sigma}, \mathrm{e}_{k}, a^{\sigma}\right), \ldots, t_{r}\left(\left(b^{\sigma}\right)^{\sigma}, \mathrm{e}_{k}, a^{\sigma}\right), \ldots\right) \\
& =q\left(a^{\sigma}, \ldots, t_{r}\left(b^{\sigma}, \mathrm{e}_{k}, a^{\sigma}\right), \ldots, t_{k}\left(b^{\sigma}, \mathrm{e}_{k}, a^{\sigma}\right), \ldots\right) \\
& =a^{\sigma} \cap_{k} b^{\sigma} \text {. }
\end{aligned}
$$

\subsection{A general representation theorem for right-handed SBAs}

In this section we show that, for every $n \geq 3$, there is a representation of an arbitrary right-handed SBA within the skew $i$-reduct of a suitable $n \mathrm{BA}$ of $n$-partitions (described in Example 3.11). The theorem also provides a new proof that every SBA can be embedded into a SBA with a maximal class (see Proposition 4.7).

Theorem 4.13. Let $n \geq 3$. Then every right-handed SBA can be embedded into the skew $i$-reduct $S_{i}(\mathbf{A})$ of a suitable nBA $\mathbf{A}$ of n-partitions.

Proof. (a) By [17, Corollary 1.14] every right-handed SBA can be embedded into an algebra of partial functions with codomain the set $\{1,2\}$ (see Example 2.15), where $0=\emptyset$ is the empty function, $f \wedge g=\left.g\right|_{G \cap F}, f \vee g=\left.f \cup g\right|_{G \cap \bar{F}}$ and $g \backslash f=\left.g\right|_{G \cap \bar{F}}$ (with $F, G$ and $H$ the domains of the functions $f, g, h$, respectively).

(b) By Corollary 3.14 every $n \mathrm{BA}$ is isomorphic to a $n \mathrm{BA}$ of $n$-partitions of a suitable set $I$ (see Examples 3.3 and 3.11). If $P=\left(P_{1}, \ldots, P_{n}\right)$ and $Q=\left(Q_{1}, \ldots, Q_{n}\right)$ are $n$-partitions of $I$, then

$$
\begin{aligned}
P \wedge_{i} Q & =t_{i}\left(P, Q, \mathrm{e}_{i}\right)=q\left(P, Q / \bar{i}, \mathrm{e}_{i} / i\right) \\
& =\left(\overline{P_{i}} \cap Q_{1}, \ldots, P_{i} \cup\left(\overline{P_{i}} \cap Q_{i}\right), \ldots, \overline{P_{i}} \cap Q_{n}\right) .
\end{aligned}
$$


The other operations can be similarly defined.

(c) We define an injective function * between the set of partial functions from a set $I$ into $\{1,2\}$ and the set of $n$-partitions of $I$. If $f: I \rightarrow\{1,2\}$ is a partial function, then $f^{*}=\left(P_{1}, \ldots, P_{n}\right)$ is the following $n$-partition of $I: P_{1}=f^{-1}(1), P_{2}=f^{-1}(2)$, $P_{i}=I \backslash \operatorname{dom}(f)$ and $P_{k}=\emptyset$ for any $k \neq 1,2, i$.

(d) The map * preserves the meet. Let $f: F \rightarrow\{1,2\}$ and $g: G \rightarrow\{1,2\}(F, G \subseteq I)$ be functions. Then we derive $(f \wedge g)^{*}=f^{*} \wedge_{i} g^{*}$ as follows:

$$
\begin{aligned}
f^{*} & =\left(f^{-1}(1), f^{-1}(2), \emptyset, \ldots, \emptyset, \bar{F}, \emptyset, \ldots, \emptyset\right) \\
g^{*} & =\left(g^{-1}(1), g^{-1}(2), \emptyset, \ldots, \emptyset, \bar{G}, \emptyset, \ldots, \emptyset\right) \\
(f \wedge g)^{*} & =\left(\left.g\right|_{G \cap F}\right)^{*} \\
& =\left(F \cap g^{-1}(1), F \cap g^{-1}(2), \emptyset, \ldots, \emptyset, \bar{G} \cup \bar{F}, \emptyset, \ldots, \emptyset\right) \\
& =\left(F \cap g^{-1}(1), F \cap g^{-1}(2), \emptyset \ldots, \bar{F} \cup(F \cap \bar{G}), \emptyset, \ldots, \emptyset\right) \\
& =f^{*} \wedge_{i} g^{*} .
\end{aligned}
$$

Similarly for the other operations.

\section{Skew star algebras}

The skew reducts of a $n \mathrm{BA}$ are so deeply related that they allow us to recover the full structure of the $n \mathrm{BA}$. It is worthwhile introducing a new variety of algebras, called skew star algebras, equationally axiomatising $n$ isomorphic SBAs and their relationships. In the main result of this section we prove that the variety of skew star algebras is term equivalent to the variety of $n \mathrm{BAs}$.

By Lemma 4.4(2) we have that the identity

$$
q\left(x, y_{1}, \ldots, y_{n}\right)=t_{1}\left(x, t_{2}\left(x, t_{3}\left(x, \ldots t_{n-1}\left(x, y_{n}, y_{n-1}\right) \ldots, y_{3}\right), y_{2}\right), y_{1}\right)
$$

holds in every $n \mathrm{BA}$. It follows that

$$
t_{n}(x, y, z)=t_{1}\left(x, t_{2}\left(x, t_{3}\left(x, \ldots t_{n-1}(x, z, y) \ldots, y\right), y\right), y\right),
$$

so that $t_{n}$ is term definable by the remaining $t_{i}(1 \leq i \leq n-1)$. This is one of the reasons for introducing $n-1$ (and not $n$ ) ternary operators in the following definition. Another reason is technical simplification.

Definition 5.1. An algebra $\mathbf{B}=\left(B, t_{1}, \ldots, t_{n-1}, 0_{1}, \ldots, 0_{n}\right)$, where $t_{i}$ is ternary and $0_{j}$ is a nullary operator, is called a skew star algebra if the following conditions hold, for every $1 \leq i, k \leq n-1$ and $1 \leq j \leq n$ :

(N0) $\left(B, t_{i}, 0_{i}\right)$ is a SRCA.

(N1) $t_{i}\left(0_{j}, y, z\right)=y(i \neq j)$.

(N2) $t_{1}\left(x, t_{2}\left(x, t_{3}\left(x,\left(\ldots t_{n-1}\left(x, 0_{n}, 0_{n-1}\right) \ldots\right), 0_{3}\right), 0_{2}\right), 0_{1}\right)=x$.

(N3) $t_{i}\left(x, t_{k}(x, y, z), t_{k}(x, u, w)\right)=t_{k}\left(x, t_{i}(x, y, u), t_{i}(x, z, w)\right)(i \neq k)$.

(N4) $t_{i}\left(x, t_{k}(x, y, z), u\right)=t_{k}\left(x, t_{i}(x, y, u), z\right)(i \neq k)$. 
(N5) $t_{i}(x,-,-)$ is a homomorphism of the algebra $\left(B, t_{k}, 0_{k}\right) \times\left(B, t_{k}, 0_{k}\right)$ into $\left(B, t_{k}, 0_{k}\right)$ :

$$
t_{i}\left(x, t_{k}\left(y_{1}, y_{2}, y_{3}\right), t_{k}\left(z_{1}, z_{2}, z_{3}\right)\right)=t_{k}\left(t_{i}\left(x, y_{1}, z_{1}\right), t_{i}\left(x, y_{2}, z_{2}\right), t_{i}\left(x, y_{3}, z_{3}\right)\right) .
$$

Skew star algebras constitute a variety of algebras.

The identities characterising skew star algebras deserve some explanation. Let $\mathbf{B}_{i}=$ $\left(B, t_{i}, 0_{i}\right)(i=1, \ldots, n-1)$ be a family of SRCAs having the same universe $B$ and such that $0_{1}, \ldots, 0_{n-1}$ are distinct elements of $B$. Let $0_{n}$ be another element of $B$ distinct from $0_{1}, \ldots, 0_{n-1}$. Let $\mathbf{B}=\left(B, t_{1}, \ldots, t_{n-1}, 0_{1}, \ldots, 0_{n}\right)$ be the algebra collecting the basic operations of the algebras $\mathbf{B}_{i}$ and the constant $0_{n}$. Roughly speaking, the structure of an $n \mathrm{BA}$ on $\mathbf{B}$ with respect to the term operation $q_{t}$, defined by

$$
q_{t}\left(x, y_{1}, \ldots, y_{n}\right):=t_{1}\left(x, t_{2}\left(x, t_{3}\left(x, \ldots t_{n-1}\left(x, y_{n}, y_{n-1}\right) \ldots, y_{3}\right), y_{2}\right), y_{1}\right),
$$

can be recovered from the cluster of SRCAs $\mathbf{B}_{i}$ if (N1) - (N5) hold:

(i) (N1) implies that $\mathbf{B}$ is a $n \mathrm{CA}$ with respect to the operation $q_{t}$.

(ii) Since $\mathbf{B}_{i}$ is a SRCA, then, for every $b \in B$, the function $t_{i}(b,-,-)$ satisfies conditions (D1) and (D2) of Definition 2.2. Then, axiom (N5) implies that, for every $b \in B$, the binary functions $t_{1}(b,-,-), \ldots, t_{n-1}(b,-,-)$ are 2-ary decomposition operators of the $n \mathrm{CA} \mathbf{B}$.

(iii) (N3) means that the decomposition operators $t_{1}(b,-,-), \ldots, t_{n-1}(b,-,-)$ are pairwise commuting. Hence, by Proposition 2.4 and by Proposition 2.5 the $n$-ary operator $q_{t}(b,-, \ldots,-)$ (see (5.1) above) is a $n$-ary decomposition operator of the $n$ CA B.

(iv) (N2) implies that the factor element $b$ satisfies the identity $q_{t}\left(b, 0_{1}, \ldots, 0_{n}\right)=b$. Then $b$ is a $n$-central element of the $n \mathrm{CA} \mathbf{B}$, for every $b \in B$. We conclude that axioms (N1), (N2), (N3) and (N5) collectively imply that $\mathbf{B}$ is a $n$ BA (w.r.t. $q_{t}$ ).

(v) Axiom (N4) is used to recover the ternary operations $t_{i}(1 \leq i \leq n-1)$ from $q_{t}$, i.e., $t_{i}(a, b, c)=q_{t}(a, b / \bar{i}, c / i)$.

We now are going to prove that the variety of skew star algebras and of $n$ BAs are term equivalent. Consider the following correspondence between the algebraic similarity types of $n$ BAs and of skew star algebras.

- Beginning on the $n \mathrm{BA}$ side: $t_{i}(x, y, z):=q(x, y / \bar{i}, z / i)(1 \leq i \leq n-1)$ and $0_{j}:=\mathrm{e}_{j}(1 \leq j \leq n)$.

- Beginning on the skew star algebra side:

$$
\begin{aligned}
q_{t}\left(x, y_{1}, \ldots, y_{n}\right) & :=t_{1}\left(x, t_{2}\left(x, t_{3}\left(x, \ldots t_{n-1}\left(x, y_{n}, y_{n-1}\right) \ldots, y_{3}\right), y_{2}\right), y_{1}\right) ; \\
\mathrm{e}_{j} & :=0_{j} .
\end{aligned}
$$

If $\mathbf{B}$ is a skew star algebra, then $\mathbf{B}^{\bullet}=\left(B ; q_{t}, \mathrm{e}_{1}, \ldots, \mathrm{e}_{n}\right)$ denotes the corresponding algebra in the similarity type of $n \mathrm{BAs}$.

Similarly, if $\mathbf{A}$ is a $n \mathbf{B A}$, then $\mathbf{A}^{*}=\left(A ; t_{1}, \ldots, t_{n-1}, 0_{1}, \ldots, 0_{n}\right)$ denotes the corresponding algebra in the similarity type of skew star algebras.

It is not difficult to prove the following theorem. 
Theorem 5.2. The above correspondences define a term equivalence between the varieties of $n B A s$ and of skew star algebras. More precisely,

(i) If $\mathbf{A}$ is a $n B A$, then $\mathbf{A}^{*}$ is a skew star algebra;

(ii) If $\mathbf{B}$ is a skew star algebra, then $\mathbf{B}^{\bullet}$ is a $n B A$;

(iii) $\left(\mathbf{A}^{*}\right)^{\bullet}=\mathbf{A}$;

(iv) $\left(\mathbf{B}^{\bullet}\right)^{*}=\mathbf{B}$.

Proof. (i): (N0) derives from Proposition 4.6, while (N1) comes from Lemma 4.4(1). (N2) follows from

$$
t_{1}\left(x, t_{2}\left(x, t_{3}\left(x, \ldots t_{n}\left(x, 0_{n-1}, 0_{n}\right) \ldots, 0_{3}\right), 0_{2}\right), 0_{1}\right)=_{(\mathrm{B} 2)} q\left(x, 0_{1}, \ldots, 0_{n}\right)=_{(\mathrm{B} 4)} x .
$$

(N3) is a consequence of Lemma 4.11(1). For (N4) we apply Lemma 4.11(2). (N5) follows from (B3).

(ii): (B0) derives from (N0) and (N1). By (N0) and (N5), $t_{i}(x,-,-)(1 \leq i \leq n)$ is a decomposition operator on $\mathbf{B}$. Then, for every $b \in B, q_{t}(b,-, \ldots,-)$ is a $n$-ary decomposition operator on $\mathbf{B}^{\bullet}$ (i.e., (B1) - (B3) hold), because commuting decomposition operators are closed under composition (see [21], Proposition 2.4 and Proposition 2.5).

(B4) is a consequence of (N2).

(iii): Let $\mathbf{A}$ be a $n$ BA. Since $t_{i}(x, y, z)=q(x, y / \bar{i}, z / i)$, then by (B2) we have:

$$
\begin{aligned}
q_{t}\left(x, y_{1}, \ldots, y_{n}\right) & =t_{1}\left(x, t_{2}(x, \ldots), y_{1}\right) \\
& =q\left(x, y_{1}, t_{2}(x, \ldots), \ldots, t_{2}(x, \ldots)\right) \\
& =q\left(x, y_{1}, y_{2}, t_{3}(x, \ldots), \ldots, t_{3}(x, \ldots)\right) \\
& \vdots \\
& =q\left(x, y_{1}, \ldots, y_{n}\right) .
\end{aligned}
$$

(iv): Let $\mathbf{B}=\left(B, t_{1}, \ldots, t_{n-1}, 0_{1}, \ldots, 0_{n}\right)$ be a skew star algebra. The conclusion $\left(\mathbf{B}^{\bullet}\right)^{*}=\mathbf{B}$ follows because by (N4) we obtain that $t_{i}(x, y, z)=q_{t}(x, y / \bar{i}, z / i)$ for every $1 \leq i \leq n-1$.

\section{Multideals}

The notion of ideal plays an important role in order theory and universal algebra. Ideals, filters and congruences are interdefinable in Boolean algebras. For every Boolean ideal $I$, we have that $a \in I$ if and only if $\neg a \in \neg I$ if and only if $a \theta_{I} 0$ if and only if $\neg a \theta_{I} 1$. In the case of $n$ BAs, the couple $(I, \neg I)$ is replaced by a $n$-tuple $\left(I_{1}, \ldots, I_{n}\right)$ satisfying some compatibility conditions that extend in a conservative way those of the Boolean case.

Definition 6.1. Let $\mathbf{A}$ be a $n \mathrm{BA}$. A multideal is a $n$-partition $\left(I_{1}, \ldots, I_{n}\right)$ of a subset $I$ of $A$ such that

(m1) $\mathrm{e}_{k} \in I_{k}$;

(m2) $a \in I_{r}, b \in I_{k}$ and $c_{1}, \ldots, c_{n} \in A$ imply $q\left(a, c_{1}, \ldots, c_{r-1}, b, c_{r+1}, \ldots, c_{n}\right) \in I_{k}$;

(m3) $a \in A$ and $c_{1}, \ldots, c_{n} \in I_{k}$ imply $q\left(a, c_{1}, \ldots, c_{n}\right) \in I_{k}$. 
The set $I$ is called the carrier of the multideal. An ultramultideal of $\mathbf{A}$ is a multideal whose carrier is $A$.

The following lemma, whose proof is straightforward, shows the appropriateness of the notion of multideal. In Section 7 we show that there exists a bijective correspondence between multideals and congruences.

Lemma 6.2. If $\theta$ is a proper congruence on a $n B A \mathbf{A}$, then $I(\theta)=\left(\mathrm{e}_{1} / \theta, \ldots, \mathrm{e}_{n} / \theta\right)$ is $a$ multideal of $\mathbf{A}$.

Multideals extend to the $n$-ary case the fundamental notions of Boolean ideal and filter, as shown in the following proposition.

Recall from [23] that a $2 \mathrm{BA} \mathbf{A}=\left(A, q, \mathrm{e}_{1}, \mathrm{e}_{2}\right)$ is term equivalent to the Boolean algebra $\mathbf{A}^{*}=(A, \wedge, \vee, \neg, 0,1)$, where $0=\mathrm{e}_{2}, 1=\mathrm{e}_{1}, x \wedge y=q(x, y, 0), x \vee y=q(x, 1, y)$, $\neg x=q(x, 0,1)$. We remind the reader here how $q$ is recovered from the Boolean algebra operations: $q(x, y, z)=(x \wedge y) \vee(\neg x \wedge z)$.

Proposition 6.3. Let $\mathbf{A}$ be a $2 B A$, and $I_{1}, I_{2} \subseteq A$. Then $\left(I_{1}, I_{2}\right)$ is a multideal of $\mathbf{A}$ if and only if $I_{2}$ is an ideal of $\mathbf{A}^{*}$, and $I_{1}=\neg I_{2}$ is the filter associated to $I_{2}$ in $\mathbf{A}^{*}$.

Proof. If $\left(I_{1}, I_{2}\right)$ is a multideal, then $0=\mathrm{e}_{2} \in I_{2}$ and $1=\mathrm{e}_{1} \in I_{1}$. Moreover, if $a, b \in I_{2}$ then $a \vee b=q(a, 1, b) \in I_{2}$ by (m2), and if $a \in I_{2}$ and $b \in A$, then $b \wedge a=q(b, a, 0) \in I_{2}$ by (m3). It follows that $I_{2}$ is a Boolean ideal. By $(\mathrm{m} 2)$ and the definition of $\neg$ we have $I_{1} \supseteq\left\{\neg a \mid a \in I_{2}\right\}$ and $I_{2} \supseteq\left\{\neg a \mid a \in I_{1}\right\}$. Then from $\neg \neg a=a$ it follows that $I_{1}=\left\{\neg a \mid a \in I_{2}\right\}$.

Conversely, if $I_{2}$ is a Boolean ideal of $\mathbf{A}^{*}$ and $I_{1}=\neg I_{2}$, then the condition (m1) is clearly satisfied. Concerning (m2), it is worth noticing that $q(a, c, b)=(a \wedge c) \vee(\neg a \wedge b)$. Then if $a \in I_{2}, b \in I_{1}, c \in A$ (for instance, the other 3 cases being similar to this one), we have that $\neg a \in I_{1}$, so that $\neg a \wedge b \in I_{1}$ and we conclude that $(a \wedge c) \vee(\neg a \wedge b)=$ $q(a, c, b) \in I_{1}$. Concerning (m3), if $a, b \in I_{2}$ and $c \in A$ then $c \wedge a, \neg c \wedge b \in I_{2}$, hence $(c \wedge a) \vee(\neg c \wedge b)=q(c, a, b) \in I_{2}$. If $a, b \in I_{1}$ and $c \in A$, then

$$
\begin{aligned}
(c \wedge a) \vee(\neg c \wedge b) & \geq(c \wedge(a \wedge b)) \vee(\neg c \wedge(a \wedge b)) \\
& =(c \vee \neg c) \wedge(a \wedge b)=a \wedge b \in I_{1},
\end{aligned}
$$

so that $(c \wedge a) \vee(\neg c \wedge b)=q(c, a, b) \in I_{1}$.

In the $n$-ary case, multideals of $\mathbf{A}$ may be characterised as $n$-tuples of ideals in the skew $i$-reducts $S_{i}(\mathbf{A})$ of $\mathbf{A}$, satisfying the conditions expressed in the following proposition.

Proposition 6.4. Let $\mathbf{A}$ be a $n B A$ and $\left(I_{1}, \ldots, I_{n}\right)$ be a n-partition of a subset $I$ of $A$. Then $\left(I_{1}, \ldots, I_{n}\right)$ is a multideal if and only if the following conditions are satisfied:

(II) $\mathrm{e}_{r} \in I_{r}$;

(I2) $a \in I_{r}, b \in I_{k}$ and $c \in A$ imply $t_{r}(a, c, b) \in I_{k}$;

(I3) $a, b \in I_{r}$ and $c \in A$ imply $t_{k}(c, a, b) \in I_{r}$, for all $k$.

Proof. Showing that a multideal satisfies (I1), (I2) and (I3) is straightforward. A $n$-partition satisfying (I1), (I2) and (I3), trivially verifies (m1). Concerning (m2), let us suppose that 
$a \in I_{r}, b \in I_{k}$ and $c_{1}, \ldots, c_{n} \in A$. In order to show that $q\left(a, c_{1}, \ldots, c_{r-1}, b, c_{r+1}, \ldots\right.$, $\left.c_{n}\right) \in I_{k}$, we apply Lemma 4.11(4):

$$
\left.q\left(x, c_{\sigma 1}, \ldots, c_{\sigma n}\right)=t_{\tau 1}\left(x, t_{\tau 2}\left(x, t_{\tau 3}\left(x, \ldots t_{\tau n}\left(x, c_{\tau n}, c_{\sigma \tau n}\right) \ldots, c_{\sigma \tau 3}\right)\right), c_{\sigma \tau 2}\right), c_{\sigma \tau 1}\right)
$$

in the case $\sigma=\mathrm{Id}, \tau=(1 r)$, and we get

$$
q\left(a, c_{1}, \ldots, c_{r-1}, b, c_{r+1}, \ldots, c_{n}\right)=t_{r}\left(a, t_{2}\left(a, \ldots, c_{2}\right), b\right) \in I_{k}, \quad \text { by (I2). }
$$

Concerning (m3), let $a_{1}, \ldots, a_{n} \in I_{k}$ and $b \in A$. By Lemma 4.4(2) we have

$$
q\left(b, a_{1}, \ldots, a_{n}\right)=t_{1}\left(b, t_{2}\left(b, t_{3}\left(b, \ldots t_{n-1}\left(b, a_{n}, a_{n-1}\right) \ldots, a_{3}\right), a_{2}\right), a_{1}\right) .
$$

By applying (I3) $n$ times, we conclude that $q\left(b, a_{1}, \ldots, a_{n}\right) \in I_{k}$, since $t_{n-1}\left(b, a_{n}\right.$, $\left.a_{n-1}\right) \in I_{k}$, hence $t_{n-2}\left(b, t_{n-1}\left(b, a_{n}, a_{n-1}\right), a_{n-2}\right) \in I_{k}$, and so on.

By using the characterisation of Proposition 6.4 it is easy to see that the components of a multideal are ideals of the SBA corresponding to their index.

Recall from Section 2.4 the notion of an ideal of a SBA.

Corollary 6.5. If $\left(I_{1}, \ldots, I_{n}\right)$ is a multideal of a $n B A \mathbf{A}$ and $1 \leq i \leq n$, then $I_{i}$ is an ideal of the skew $i$-reduct $S_{i}(\mathbf{A})=\left(A, \wedge_{i}, \vee_{i}, \backslash_{i}, \mathrm{e}_{i}\right)$.

Proof. Since $S_{i}(\mathbf{A})$ is right-handed, a non empty set $K \subseteq A$ is an ideal of $S_{i}(\mathbf{A})$ if and only if, for all $a, b \in K$ and $c \in A, a \vee_{i} b \in K$ and $a \wedge_{i} c \in K$ (see Section 2.4). Given $a, b \in I_{i}$ and $c \in A$, we have $a \vee_{i} b=t_{i}(a, a, b) \in I_{i}$ and $a \wedge_{i} c=t_{i}\left(a, c, \mathrm{e}_{i}\right) \in I_{i}$, by using in both cases condition (I2) of Proposition 6.4 (notice that $\mathrm{e}_{i} \in I_{i}$, by (I1)).

Lemma 6.6. The carrier I of a multideal $\left(I_{1}, \ldots, I_{n}\right)$ of a nBA A is a subalgebra of $\mathbf{A}$.

Proof. The constants $\mathrm{e}_{1}, \ldots, \mathrm{e}_{n}$ belong to $I$ by (m1). If $a \in I_{r}$ and $b \in I_{k}$, then $q\left(a, c_{1}\right.$, $\left.\ldots, c_{r-1}, b, c_{r+1}, \ldots, c_{n}\right) \in I_{k}$, for all $c_{1}, \ldots, c_{n} \in A$, by (m2). Hence $I$ is a subalgebra of $\mathbf{A}$.

Any component $I_{i}$ of a multideal $\left(I_{1}, \ldots, I_{n}\right)$ determines the multideal completely, as shown in the following lemma.

Lemma 6.7. If $\left(I_{1}, \ldots, I_{n}\right)$ is a multideal of a $n B A \mathbf{A}$, then $I_{k}=I_{r}^{(r k)}$ for all $r, k$.

Proof. Let $a \in I_{r}$. Then $a^{(r k)}=t_{r}\left(a, t_{k}\left(a, a, \mathrm{e}_{r}\right), \mathrm{e}_{k}\right) \in I_{k}$ by Lemma 4.11(5) and Proposition 6.4(I2). Then we have

$$
I_{r}^{(r k)} \subseteq I_{k} ; \quad I_{k}^{(r k)} \subseteq I_{r}
$$

The conclusion follows because $\left(a^{(r k)}\right)^{(r k)}=a$, by Lemma 4.11(6) and (B4).

Multideals are closed under arbitrary nonempty componentwise intersection. The minimum multideal is the sequence $\left(\left\{\mathrm{e}_{k}\right\}\right)_{k \in \hat{n}}$. Given a $n \mathrm{BA} \mathbf{A}$, and $A_{1}, \ldots, A_{n} \subseteq A$, let us consider the set $\mathcal{A}$ of multideals containing $\left(A_{1}, \ldots, A_{n}\right)$. The ideal closure of $\left(A_{1}, \ldots, A_{n}\right)$ is the componentwise intersection of the elements of $\mathcal{A}$, if $\mathcal{A} \neq \emptyset$. Otherwise, the ideal closure of $\left(A_{1}, \ldots, A_{n}\right)$ is the constant $n$-tuple $I^{\top}=(A, \ldots, A)$, that we consider as a degenerate multideal, by a small abuse of terminology.

As a matter of fact, $I^{\top}$ is the only degenerate multideal. 
Lemma 6.8. Let $\mathbf{A}$ be a $n B A$ and $I=\left(I_{1}, \ldots, I_{n}\right)$ be a tuple of subsets of $A$ satisfying the closure properties of Definition 6.1. The following are equivalent:

(i) there exist $a \in A$ and $r \neq k$ such that $a \in I_{r} \cap I_{k}$.

(ii) there exist $r \neq k$ such that $\mathrm{e}_{k} \in I_{r}$.

(iii) $I=I^{\top}$.

Proof. (i) $\Rightarrow$ (ii): Since $a \in I_{k}$, by Lemma 6.7 we have that $a^{(r k)} \in I_{r}$. By Definition 6.1(m2), we conclude that $q\left(a, \mathrm{e}_{k} / \bar{r}, a^{(r k)} / r\right)={ }_{(\mathrm{B} 2)} q\left(a, \mathrm{e}_{k}, \ldots, \mathrm{e}_{k}\right)=\mathrm{e}_{k} \in I_{r}$.

(ii) $\Rightarrow$ (iii): Given $b \in A$, we have $b=q\left(\mathrm{e}_{k}, b / \bar{r}, \mathrm{e}_{r} / r\right) \in I_{r}$ by Definition $6.1(\mathrm{~m} 2)$. Hence $I_{r}=A$ and the result follows from Lemma 6.7 since $A^{(r k)}=A$ for all $1 \leq k \leq n$. (iii) $\Rightarrow$ (i): Trivial.

\section{The relationship between multideals and congruences}

For any congruence $\theta$ on a $n \mathrm{BA}$, the equivalence classes $\mathrm{e}_{i} / \theta$ form a multideal (see Lemma 6.2), exactly as in the Boolean case where $0 / \theta$ is an ideal and $1 / \theta$ the corresponding filter. Conversely, in the Boolean case, any ideal $I$ (resp. filter $F$ ) defines the congruence $x \theta_{I} y \Leftrightarrow x \oplus y \in I$ (resp. $\left.x \theta_{F} y \Leftrightarrow x \leftrightarrow y \in F\right)$, where $x \oplus y=(x \wedge \neg y) \vee(\neg x \wedge y)$ and $x \leftrightarrow y=(\neg x \vee y) \wedge(x \vee \neg y)$. Rephrasing this latter correspondence in the $n$-ary case is a bit more complicated.

\subsection{The Boolean algebra of coordinates}

Let $\mathbf{A}$ be a $n \mathrm{BA}, a \in A$ and $i \in \hat{n}$. We consider the factor congruence $\theta_{a}^{i}=\theta\left(a, \mathrm{e}_{i}\right)=$ $\left\{(x, y): t_{i}(a, x, y)=x\right\}$ generated by $a$. By Lemma 6.2 the tuple $\left(\mathrm{e}_{1} / \theta_{a}^{i}, \ldots, \mathrm{e}_{n} / \theta_{a}^{i}\right)$ is a multideal of $\mathbf{A}$.

We recall that $\preccurlyeq_{\mathcal{R}}^{i}$ and $\leq^{i}$ denote the preorder and the partial order of the SBA $S_{i}(\mathbf{A})=$ $\left(A, \wedge_{i}, \vee_{i}, \backslash i, \mathrm{e}_{i}\right)$, respectively (see Section 2.4).

Lemma 7.1. $\mathrm{e}_{i} / \theta_{a}^{i}=\left\{b \in A: b \preccurlyeq_{\mathcal{R}}^{i} a\right\}$.

Proof. By definition of $\theta_{a}^{i}$, we have $b \theta_{a}^{i} c$ iff $t_{i}(a, b, c)=b$. Then $b \in \mathrm{e}_{i} / \theta_{a}^{i}$ iff $b=$ $t_{i}\left(a, b, \mathrm{e}_{i}\right)=a \wedge_{i} b$ iff $b \preccurlyeq_{\mathcal{R}}^{i} a$ (by definition of $\preccurlyeq_{\mathcal{R}}^{i}$ ).

The following proposition is a consequence of [9, Proposition 4.15].

\section{Proposition 7.2.}

(i) The set $\mathrm{e}_{i} / \theta_{a}^{i}$ is a subalgebra of the right Church $i$-reduct $\left(A, t_{i}, \mathrm{e}_{i}\right)$.

(ii) The algebra $\left(\mathrm{e}_{i} / \theta_{a}^{i}, t_{i}, \mathrm{e}_{i}, a\right)$ is a $2 C A$.

(iii) The set $\downarrow_{i} a=\left\{b: b \leq^{i} a\right\}$ is the Boolean algebra of 2-central elements of $\left(\mathrm{e}_{i} / \theta_{a}^{i}, t_{i}, \mathrm{e}_{i}, a\right)$.

Proof. (i): Let $b, c, d \in \mathrm{e}_{i} / \theta_{a}^{i}$. Then $b \theta_{a}^{i} \mathrm{e}_{i}, c \theta_{a}^{i} \mathrm{e}_{i}$ and $d \theta_{a}^{i} \mathrm{e}_{i}$. By applying the properties of the congruences, we derive $t_{i}(b, c, d) \theta_{a}^{i} t_{i}\left(\mathrm{e}_{i}, \mathrm{e}_{i}, \mathrm{e}_{i}\right)=\mathrm{e}_{i}$.

(ii): By $t_{i}(a, b, c)=b$ and $t_{i}\left(\mathrm{e}_{i}, b, c\right)=c$, for every $b, c \in \mathrm{e}_{i} / \theta_{a}^{i}$.

(iii): By Lemma 4.5(i) $b$ is a factor element for every $b \in \mathrm{e}_{i} / \theta_{a}^{i}$. Then $b$ is 2-central iff $t_{i}\left(b, a, \mathrm{e}_{i}\right)=b$ iff $b \wedge_{i} a=b$ iff $b \leq^{i} a$, because $a \wedge_{i} b=t_{i}\left(a, b, \mathrm{e}_{i}\right)=b$ for all $b \in \mathrm{e}_{i} / \theta_{a}^{i}$. 
Notice that $a$ is maximal (w.r.t. $\leq^{i}$ ) because, if $a \leq^{i} b \in \mathrm{e}_{i} / \theta_{a}^{i}$, then $a=a \wedge_{i} b=$ $t_{i}\left(a, b, \mathrm{e}_{i}\right)=b$.

We now specialise the above construction to the case $a=\mathrm{e}_{j}$ for a given $j \neq i$.

Definition 7.3. Let $\mathbf{A}$ be a $n \mathrm{BA}$ and $i \neq j$. The Boolean centre of $\mathbf{A}$, denoted by $\mathbf{B}_{i j}$, is the Boolean algebra of 2-central elements of the 2CA $\left(A, t_{i}, \mathrm{e}_{i}, \mathrm{e}_{j}\right)$.

By Proposition 7.2(iii) the carrier set of $\mathbf{B}_{i j}$ is the set $\downarrow_{i} \mathrm{e}_{j}=\left\{b \in A: b \leq{ }^{i} \mathrm{e}_{j}\right\}$ and we call Boolean any element of $\mathbf{B}_{i j}$.

Remark 7.4. The Boolean algebra $\mathbf{B}_{i j}$ was defined in [6] in a different but equivalent way (see [6, Section 6.1, Lemma 7(iii)]).

Lemma 7.5. Let $\mathbf{A}$ be a $n B A, \mathbf{B}_{i j}$ be the Boolean centre of $\mathbf{A}, S_{i}(\mathbf{A})=\left(A, \wedge_{i}, \vee_{i}, \backslash_{i}, \mathrm{e}_{i}\right)$ be the skew $i$-reduct of $\mathbf{A}$, and $i \neq j$. Then, for all $b, c \in B_{i j}$, we have $b \vee_{i} c=t_{i}\left(b, \mathrm{e}_{j}, c\right)$.

Proof. $b \vee_{i} c=t_{i}(b, b, c)={ }_{\left(b \leq{ }^{i} \mathrm{e}_{j}\right)} t_{i}\left(b, t_{i}\left(b, \mathrm{e}_{j}, \mathrm{e}_{i}\right), c\right)={ }_{(\mathrm{B} 2)} t_{i}\left(b, \mathrm{e}_{j}, c\right)$.

By Lemma 7.5 and by [23] the Boolean operations on $\mathbf{B}_{i j}$ are $\wedge_{i}, \vee_{i}, \neg_{i j}$, where $\wedge_{i}, \vee_{i}$ are the corresponding operation of $S_{i}(\mathbf{A})$ restricted to $\mathbf{B}_{i j}$, and $\neg_{i j}(b)=t_{i}\left(b, \mathrm{e}_{i}, \mathrm{e}_{j}\right)$ for every $b \in B_{i j}$.

In [6] a representation theorem is proved, showing that any given $n \mathrm{BA} \mathbf{A}$ can be embedded into the $n \mathrm{BA}$ of the $n$-central elements of the Boolean vector space $B_{i j} \times \ldots \times B_{i j}=$ $\left(B_{i j}\right)^{n}$ (see Example 3.2). The proof of this result makes an essential use of the notion of coordinates of elements of $\mathbf{A}$, that are $n$-tuples of elements of $\left(B_{i j}\right)^{n}$, codifying the elements of $\mathbf{A}$ as linear combinations (see Lemma 7.12(5)). In this paper, the notion of coordinate is again a central one, being used to define the congruence associated to a multideal. In order to highlight their relationship with the skew reducts of $\mathbf{A}$, here we define the coordinates in terms of the operations $t_{k}$.

Definition 7.6. The coordinates of $a \in A$ are the elements $a_{\mathrm{k}}=t_{k}\left(a, \mathrm{e}_{i}, \mathrm{e}_{j}\right)$, for $1 \leq k \leq n$.

Notice that $a_{\mathrm{k}} \in B_{i j}$ for every $1 \leq k \leq n$, because $a_{\mathrm{k}} \leq{ }^{i} \mathrm{e}_{j}$ :

$$
a_{\mathrm{k}} \wedge_{i} \mathrm{e}_{j}=t_{i}\left(t_{k}\left(a, \mathrm{e}_{i}, \mathrm{e}_{j}\right), \mathrm{e}_{j}, \mathrm{e}_{i}\right)={ }_{(\mathrm{B} 3)} q\left(a, \mathrm{e}_{i} / \bar{k}, \mathrm{e}_{j} / k\right)=t_{k}\left(a, \mathrm{e}_{i}, \mathrm{e}_{j}\right)=a_{\mathrm{k}} .
$$

Lemma 7.7. Let $a, b, b_{1}, \ldots, b_{n} \in A$. We have:

(i) $a_{\mathrm{k}} \wedge_{i} a_{\mathrm{r}}=\mathrm{e}_{i}$ for all $k \neq r$.

(ii) $a_{1} \vee_{i} a_{2} \vee_{i} \cdots \vee_{i} a_{\mathrm{n}}=\mathrm{e}_{j}$

(iii) $q\left(a, b_{1}, \ldots, b_{n}\right)_{\mathrm{k}}=q\left(a,\left(b_{1}\right)_{\mathrm{k}}, \ldots,\left(b_{n}\right)_{\mathrm{k}}\right)=\left(a_{1} \wedge_{i}\left(b_{1}\right)_{\mathrm{k}}\right) \vee_{i} \cdots \vee_{i}\left(a_{\mathrm{n}} \wedge_{i}\left(b_{n}\right)_{\mathrm{k}}\right)$.

(iv) $\left(a \wedge_{i} b\right)_{\mathrm{k}}=a \wedge_{i} b_{\mathrm{k}}$, for every $k \neq i$.

(v) $a_{\mathrm{k}} \wedge_{i} a=a_{\mathrm{k}} \wedge_{i} \mathrm{e}_{k}$, for every $k \neq i$.

(vi) $a_{\mathrm{i}} \wedge_{i} a=\mathrm{e}_{i}$. 
(vii) If $a \in B_{i j}$, then

$$
a_{\mathrm{k}}= \begin{cases}\neg_{i j}(a) & \text { if } k=i \\ a & \text { if } k=j \\ \mathrm{e}_{i} & \text { otherwise }\end{cases}
$$

Proof. (i)-(vi): It is sufficient to check in the generator $\mathbf{n}$ of the variety $n$ BA, where $B_{i j}=\left\{\mathrm{e}_{i}, \mathrm{e}_{j}\right\},\left(\mathrm{e}_{r}\right)_{\mathrm{r}}=\mathrm{e}_{j}$ and $\left(\mathrm{e}_{r}\right)_{\mathrm{k}}=\mathrm{e}_{i}$ if $r \neq k$.

(vii): $(k=i)$ : By definition of $\neg_{i j}$ :

$$
a_{\mathrm{i}}=t_{i}\left(a, \mathrm{e}_{i}, \mathrm{e}_{j}\right)=\neg_{i j}(a) .
$$

$(k \neq i, j)$ :

$$
\begin{aligned}
& a_{\mathrm{k}}=t_{k}\left(a, \mathrm{e}_{i}, \mathrm{e}_{j}\right)=t_{k}\left(a \wedge_{i} \mathrm{e}_{j}, \mathrm{e}_{i}, \mathrm{e}_{j}\right)=t_{k}\left(t_{i}\left(a, \mathrm{e}_{j}, \mathrm{e}_{i}\right), \mathrm{e}_{i}, \mathrm{e}_{j}\right)=t_{i}\left(a, \mathrm{e}_{i}, \mathrm{e}_{i}\right)=\mathrm{e}_{i} . \\
& (k=j): \\
& a_{\mathrm{j}}=t_{j}\left(a, \mathrm{e}_{i}, \mathrm{e}_{j}\right)=t_{j}\left(a \wedge_{i} \mathrm{e}_{j}, \mathrm{e}_{i}, \mathrm{e}_{j}\right)=t_{j}\left(t_{i}\left(a, \mathrm{e}_{j}, \mathrm{e}_{i}\right), \mathrm{e}_{i}, \mathrm{e}_{j}\right)=t_{i}\left(a, \mathrm{e}_{j}, \mathrm{e}_{i}\right)=a .
\end{aligned}
$$

Proposition 7.8. The following conditions are equivalent for an element $a \in A$ :

(a) a is Boolean;

(b) $a \wedge_{i} \mathrm{e}_{j}=a$;

(c) $a=b_{\mathrm{k}}$, for some $b \in A$ and index $1 \leq k \leq n$;

(d) $a=a_{\mathrm{j}}$;

(e) $a_{\mathrm{k}}=\mathrm{e}_{i}$, for every $k \neq i, j$;

(f) $a=\left(a_{\mathrm{i}}\right)_{\mathrm{i}}$.

Proof. (a) $\Leftrightarrow$ (b): We have that $a \leq^{i} \mathrm{e}_{j}$ iff $a \wedge_{i} \mathrm{e}_{j}=a$ and $\mathrm{e}_{j} \wedge_{i} a=a$. The conclusion is obtained because the latter equality is trivially true.

(c) $\Rightarrow(\mathrm{b})$ :

$$
b_{\mathrm{k}} \wedge_{i} \mathrm{e}_{j}=t_{i}\left(t_{k}\left(b, \mathrm{e}_{i}, \mathrm{e}_{j}\right), \mathrm{e}_{j}, \mathrm{e}_{i}\right)={ }_{(\mathrm{B} 3)} q\left(b, \mathrm{e}_{i} / \bar{k}, \mathrm{e}_{j} / k\right)=t_{k}\left(b, \mathrm{e}_{i}, \mathrm{e}_{j}\right)=b_{\mathrm{k}} .
$$

(b) $\Rightarrow$ (d): If $a \wedge_{i} \mathrm{e}_{j}=a$, then we have:

$$
\begin{aligned}
a_{\mathrm{j}} & =t_{j}\left(a, \mathrm{e}_{i}, \mathrm{e}_{j}\right)=t_{j}\left(a \wedge_{i} \mathrm{e}_{j}, \mathrm{e}_{i}, \mathrm{e}_{j}\right)=t_{j}\left(t_{i}\left(a, \mathrm{e}_{j}, \mathrm{e}_{i}\right), \mathrm{e}_{i}, \mathrm{e}_{j}\right) \\
& ={ }_{\text {(B3) }} t_{i}\left(a, \mathrm{e}_{j}, \mathrm{e}_{i}\right)=a \wedge_{i} \mathrm{e}_{j}=a .
\end{aligned}
$$

(d) $\Rightarrow$ (c): Trivial.

(a) $\Rightarrow$ (e): By Lemma 7.7(vii).

(e) $\Rightarrow$ (d): By Lemma 7.7(ii) the join of all coordinates of $a \in A$ in $\mathbf{B}_{i j}$ is the top element $\mathrm{e}_{j}$. By hypothesis (e) we derive $a_{\mathrm{i}} \vee_{i} a_{\mathrm{j}}=\mathrm{e}_{j}$. Then, by applying the strong distributive property of $\wedge_{i}$ w.r.t. $\vee_{i}$ in the SBA $S_{i}(\mathbf{A})$, we obtain:

$$
\begin{aligned}
& a=\mathrm{e}_{j} \wedge_{i} a={ }_{(\operatorname{Lemma} 7.7(\mathrm{ii}))}\left(a_{\mathrm{i}} \vee_{i} a_{\mathrm{j}}\right) \wedge_{i} a=\left(a_{\mathrm{i}} \wedge_{i} a\right) \vee_{i}\left(a_{\mathrm{j}} \wedge_{i} a\right) \\
& ={ }_{(\operatorname{Lemma} 7.7(\mathrm{vi}))} \mathrm{e}_{i} \vee_{i}\left(a_{\mathrm{j}} \wedge_{i} a\right)=a_{\mathrm{j}} \wedge_{i} a={ }_{(\operatorname{Lemma} 7.7(\mathrm{v}))} a_{\mathrm{j}} \wedge_{i} \mathrm{e}_{j}={ }_{\left(a_{\mathrm{j}} \leq{ }^{i} \mathrm{e}_{j}\right)} a_{\mathrm{j}} .
\end{aligned}
$$

(f) $\Leftrightarrow(\mathrm{b}):\left(a_{\mathrm{i}}\right)_{\mathrm{i}}=t_{i}\left(t_{i}\left(a, \mathrm{e}_{i}, \mathrm{e}_{j}\right), \mathrm{e}_{i}, \mathrm{e}_{j}\right)={ }_{(\mathrm{B} 3)} t_{i}\left(a, \mathrm{e}_{j}, \mathrm{e}_{i}\right)=a \wedge_{i} \mathrm{e}_{j}$. Then $\left(a_{\mathrm{i}}\right)_{\mathrm{i}}=a$ iff $a \wedge_{i} \mathrm{e}_{j}=a$.

By Lemma 7.7(iv) and Proposition 7.8(d) $a \wedge_{i} b$ is a Boolean element, for every $a \in A$ and $b \in B_{i j}$. 


\subsection{The congruence defined by a multideal}

Let $\mathbf{A}$ be a $n \mathrm{BA}$ and $\mathbf{B}_{i j}$ be the Boolean centre of $\mathbf{A}$.

Lemma 7.9. Let $I$ be a multideal on A. Then $I_{*}=B_{i j} \cap I_{i}$ is a Boolean ideal and $I^{*}=B_{i j} \cap I_{j}$ is the Boolean filter complement of $I_{*}$.

Proof. Recall that, in $\mathbf{B}_{i j}$, $\mathrm{e}_{i}$ is the bottom element, $\mathrm{e}_{j}$ is the top element and $b \in B_{i j}$ iff $b \wedge_{i} \mathrm{e}_{j}=b$. We prove that $I_{*}$ is a Boolean ideal. First $\mathrm{e}_{i} \in I_{*}$. If $b, c \in I_{*}$ and $d \in B_{i j}$, then we prove that $b \vee_{i} c$ and $b \wedge_{i} d$ belong to $I_{*}$. By Proposition 6.4(I2) $b \vee_{i} c$ and $b \wedge_{i} d$ belong to $I_{i}$. Moreover, since $b, c, d \in B_{i j}$ and $\wedge_{i}, \vee_{i}$ are respectively the meet and the join of $\mathbf{B}_{i j}$, then $b \vee_{i} c$ and $b \wedge_{i} d$ also belong to $\mathbf{B}_{i j}$. We now show that $I^{*}$ is the Boolean filter complement of $I_{*}$.

( $b \in I_{*} \Rightarrow \neg_{i j} b \in I^{*}$ ): As $b \in I_{i} \cap B_{i j}$, then by Proposition 6.4(I2)

$$
\neg_{i j} b=t_{i}\left(b, \mathrm{e}_{i}, \mathrm{e}_{j}\right) \in I_{j} \cap B_{i j} .
$$

$\left(\neg i j b \in I^{*} \Rightarrow b \in I_{*}\right):$ As $t_{i}\left(b, \mathrm{e}_{i}, \mathrm{e}_{j}\right) \in I_{j}$, then

$$
b=\neg_{i j} \neg_{i j} b=t_{i}\left(t_{i}\left(b, \mathrm{e}_{i}, \mathrm{e}_{j}\right), \mathrm{e}_{i}, \mathrm{e}_{j}\right) \in I_{i} .
$$

The following lemma characterises multideals in terms of coordinates.

Lemma 7.10. Let $\left(I_{1}, \ldots, I_{n}\right)$ be a multideal on a $n B A \mathbf{A}$ and let $b \in A$. Then we have:

(a) $b \in I_{r}$ if and only if the coordinate $b_{\mathrm{r}}$ of $b$ belongs to $I_{j}$.

(b) If $b \in I_{i}$, then the coordinate $b_{\mathrm{k}}$ of $b$ belongs to $I_{i}$, for every $k \neq i$.

Proof. (a): We start with $r=i$.

$(\Rightarrow)$ : Let $b \in I_{i}$. By Proposition 6.4(I2) we have $b_{\mathrm{i}}=t_{i}\left(b, \mathrm{e}_{i}, \mathrm{e}_{j}\right) \in I_{j}$, because $b \in I_{i}$ and $\mathrm{e}_{j} \in I_{j}$.

$(\Leftarrow)$ : By hypothesis $b_{\mathrm{i}} \in I_{j}$. Then by Lemma $6.7 b_{\mathrm{i}}^{(i j)} \in I_{i}$. Now

$$
\begin{aligned}
b_{\mathrm{i}}^{(i j)} & =q\left(b_{\mathrm{i}}, \mathrm{e}_{(i j) 1}, \ldots, \mathrm{e}_{(i j) n}\right)=q\left(t_{i}\left(b, \mathrm{e}_{i}, \mathrm{e}_{j}\right), \mathrm{e}_{(i j) 1}, \ldots, \mathrm{e}_{(i j) n}\right) \\
& ={ }_{\text {(B3) }} t_{i}\left(b, \mathrm{e}_{j}, \mathrm{e}_{i}\right)=b \wedge_{i} \mathrm{e}_{j} \in I_{i} .
\end{aligned}
$$

We conclude $b \in I_{i}$ by applying Proposition 6.4(I2) to $t_{i}\left(b \wedge_{i} \mathrm{e}_{j}, b, \mathrm{e}_{i}\right)$, because $b \wedge_{i} \mathrm{e}_{j} \in I_{i}$ and $b={ }_{\left(\text {Lemma 4.4(3)) }_{3}\right.} b \wedge_{i} b={ }_{\left(b=\mathrm{e}_{j} \wedge_{i} b\right)} b \wedge_{i} \mathrm{e}_{j} \wedge_{i} b=t_{i}\left(b \wedge_{i} \mathrm{e}_{j}, b, \mathrm{e}_{i}\right)$.

We analyse $r \neq i$. Let $\sigma$ be equal to the transposition $(i r)$. By definition of $b^{\sigma}$ we derive $\left(b^{\sigma}\right)_{\mathrm{i}}=t_{i}\left(q\left(b, \mathrm{e}_{\sigma 1}, \ldots, \mathrm{e}_{\sigma n}\right), \mathrm{e}_{i}, \mathrm{e}_{j}\right)={ }_{(\mathrm{B} 3)} q\left(b, \mathrm{e}_{j} / r, \mathrm{e}_{i} / \bar{r}\right)=t_{r}\left(b, \mathrm{e}_{i}, \mathrm{e}_{j}\right)=b_{\mathrm{r}}$. Then, $b_{\mathrm{r}} \in I_{j} \Leftrightarrow b_{\mathrm{r}}=\left(b^{\sigma}\right)_{\mathrm{i}} \in I_{j} \Leftrightarrow b^{\sigma} \in I_{i} \Leftrightarrow \Leftrightarrow_{(\text {Lemma 6.7) }} b=\left(b^{\sigma}\right)^{\sigma} \in I_{r}$.

(b): By Proposition 6.4(I2), $k \neq i$ and $b \in I_{i}$ we get $b_{\mathrm{k}}=t_{k}\left(b, \mathrm{e}_{i}, \mathrm{e}_{j}\right) \in I_{i}$.

We consider the homomorphism $f_{I}: \mathbf{B}_{i j} \rightarrow \mathbf{B}_{i j} / I_{*}$ and we define on $A$ the following equivalence relation:

$$
b \theta_{I} c \Leftrightarrow \forall k . f_{I}\left(b_{\mathrm{k}}\right)=f_{I}\left(c_{\mathrm{k}}\right),
$$

where $b_{\mathrm{k}}, c_{\mathrm{k}}$ are the $k$-coordinates of $b$ and $c$, respectively (see Definition 7.6).

Proposition 7.11. $\theta_{I}$ is a congruence on $\mathbf{A}$. 
Proof. Let $a, b, c_{1}, d_{1}, \ldots, c_{n}, d_{n}$ be elements of $A$ such that $a \theta_{I} b$ and $c_{k} \theta_{I} d_{k}$, for every $k$. Then $q\left(a, c_{1}, \ldots, c_{n}\right) \theta_{I} q\left(b, d_{1}, \ldots, d_{n}\right)$ iff

$$
\forall k \cdot f_{I}\left(q\left(a, c_{1}, \ldots, c_{n}\right)_{\mathrm{k}}\right)=f_{I}\left(q\left(b, d_{1}, \ldots, d_{n}\right)_{\mathrm{k}}\right) .
$$

The conclusion follows, because $f_{I}$ is a Boolean homomorphism and

$$
\begin{aligned}
q\left(a, c_{1}, \ldots, c_{n}\right)_{\mathrm{k}} & ={ }_{(\operatorname{Lemma} 7.7(\mathrm{iii}))} q\left(a,\left(c_{1}\right)_{\mathrm{k}}, \ldots,\left(c_{n}\right)_{\mathrm{k}}\right) \\
& ={ }_{(\operatorname{Lemma} 7.7(\mathrm{iii}))}\left(a_{1} \wedge_{i}\left(c_{1}\right)_{\mathrm{k}}\right) \vee_{i} \cdots \vee_{i}\left(a_{\mathrm{n}} \wedge_{i}\left(c_{n}\right)_{\mathrm{k}}\right)
\end{aligned}
$$

We define a new term operation to be used in Theorem 7.13:

$$
x+{ }_{i} y=q\left(x, t_{i}\left(y, \mathrm{e}_{i}, \mathrm{e}_{1}\right), \ldots, t_{i}\left(y, \mathrm{e}_{i}, \mathrm{e}_{i-1}\right), y, t_{i}\left(y, \mathrm{e}_{i}, \mathrm{e}_{i+1}\right), \ldots, t_{i}\left(y, \mathrm{e}_{i}, \mathrm{e}_{n}\right)\right),
$$

where $y$ is at position $i$.

Lemma 7.12. Let $a, b \in A$ and $a_{1}, a_{2}, \ldots, a_{\mathrm{n}}$ be the coordinates of $a$. Then

(1) $a+{ }_{i} \mathrm{e}_{i}=\mathrm{e}_{i}+{ }_{i} a=a$;

(2) $a+{ }_{i} b=b+{ }_{i} a$;

(3) $a+{ }_{i} \mathrm{e}_{k}=\mathrm{e}_{k}+{ }_{i} a=a_{\mathrm{i}} \wedge_{i} \mathrm{e}_{k}(k \neq i)$.

(4) $a+{ }_{i} a=\mathrm{e}_{i}$;

(5) The value of the expression $E \equiv\left(a_{1} \wedge_{i} \mathrm{e}_{1}\right)+{ }_{i}\left(\left(a_{2} \wedge_{i} \mathrm{e}_{2}\right)+{ }_{i}\left(\cdots+{ }_{i}\left(a_{\mathrm{n}} \wedge_{i} \mathrm{e}_{n}\right)\right) \cdots\right)$ is independent of the order of its parentheses. Without loss of generality, we write $\left(a_{1} \wedge_{i} \mathrm{e}_{1}\right)+{ }_{i}\left(a_{2} \wedge_{i} \mathrm{e}_{2}\right)+{ }_{i} \cdots+{ }_{i}\left(a_{\mathrm{n}} \wedge_{i} \mathrm{e}_{n}\right)$ for the expression E. Then we have:

$$
\left(a_{1} \wedge_{i} \mathrm{e}_{1}\right)+_{i}\left(a_{2} \wedge_{i} \mathrm{e}_{2}\right)+{ }_{i} \cdots+{ }_{i}\left(a_{\mathrm{n}} \wedge_{i} \mathrm{e}_{n}\right)=a .
$$

(6) If $a$ and $b$ have the same coordinates, then $a=b$.

Proof. (1):

$$
\begin{aligned}
a+{ }_{i} \mathrm{e}_{i} & =q\left(a, t_{i}\left(\mathrm{e}_{i}, \mathrm{e}_{i}, \mathrm{e}_{1}\right), \ldots, t_{i}\left(\mathrm{e}_{i}, \mathrm{e}_{i}, \mathrm{e}_{i-1}\right), \mathrm{e}_{i}, t_{i}\left(\mathrm{e}_{i}, \mathrm{e}_{i}, \mathrm{e}_{i+1}\right), \ldots, t_{i}\left(\mathrm{e}_{i}, \mathrm{e}_{i}, \mathrm{e}_{n}\right)\right) \\
& =q\left(a, \mathrm{e}_{1}, \ldots, \mathrm{e}_{n}\right)={ }_{(\mathrm{B} 4)} a .
\end{aligned}
$$

(2):

$$
\begin{aligned}
& a+{ }_{i} b=q\left(a, t_{i}\left(b, \mathrm{e}_{i}, \mathrm{e}_{1}\right), \ldots, t_{i}\left(b, \mathrm{e}_{i}, \mathrm{e}_{i-1}\right), b, t_{i}\left(b, \mathrm{e}_{i}, \mathrm{e}_{i+1}\right), \ldots, t_{i}\left(b, \mathrm{e}_{i}, \mathrm{e}_{n}\right)\right) \\
& ={ }_{(\mathrm{B} 4)} q\left(t_{i}(b, a, a), t_{i}\left(b, \mathrm{e}_{i}, \mathrm{e}_{1}\right), \ldots, t_{i}\left(b, \mathrm{e}_{i}, \mathrm{e}_{i-1}\right)\right. \text {, } \\
& \left.q\left(b, \mathrm{e}_{1}, \ldots, \mathrm{e}_{n}\right), t_{i}\left(b, \mathrm{e}_{i}, \mathrm{e}_{i+1}\right), \ldots, t_{i}\left(b, \mathrm{e}_{i}, \mathrm{e}_{n}\right)\right) \\
& ={ }_{\text {(B3) }} q\left(b, t_{i}\left(a, \mathrm{e}_{i}, \mathrm{e}_{1}\right), \ldots, t_{i}\left(a, \mathrm{e}_{i}, \mathrm{e}_{i-1}\right)\right. \text {, } \\
& \left.q\left(a, \mathrm{e}_{1}, \ldots, \mathrm{e}_{n}\right), t_{i}\left(a, \mathrm{e}_{i}, \mathrm{e}_{i+1}\right), \ldots, t_{i}\left(a, \mathrm{e}_{i}, \mathrm{e}_{n}\right)\right) \\
& =b+{ }_{i} a \text {. }
\end{aligned}
$$

(3):

$$
\begin{aligned}
a+{ }_{i} \mathrm{e}_{k} & =q\left(a, t_{i}\left(\mathrm{e}_{k}, \mathrm{e}_{i}, \mathrm{e}_{1}\right), \ldots, t_{i}\left(\mathrm{e}_{k}, \mathrm{e}_{i}, \mathrm{e}_{i-1}\right), \mathrm{e}_{k}, t_{i}\left(\mathrm{e}_{k}, \mathrm{e}_{i}, \mathrm{e}_{i+1}\right), \ldots, t_{i}\left(\mathrm{e}_{k}, \mathrm{e}_{i}, \mathrm{e}_{n}\right)\right) \\
& ={ }_{(k \neq i)} q\left(a, \mathrm{e}_{i}, \ldots, \mathrm{e}_{i}, \mathrm{e}_{k}, \mathrm{e}_{i}, \ldots, \mathrm{e}_{i}\right)=t_{i}\left(a, \mathrm{e}_{i}, \mathrm{e}_{k}\right)=\mathrm{e}_{k}+{ }_{i} a .
\end{aligned}
$$


Moreover, $a_{\mathrm{i}} \wedge_{i} \mathrm{e}_{k}=t_{i}\left(t_{i}\left(a, \mathrm{e}_{i}, \mathrm{e}_{j}\right), \mathrm{e}_{k}, \mathrm{e}_{i}\right)=\left(\right.$ B3) $t_{i}\left(a, \mathrm{e}_{i}, \mathrm{e}_{k}\right)$.

(4):

$$
\begin{aligned}
a+{ }_{i} a & =q\left(a, t_{i}\left(a, \mathrm{e}_{i}, \mathrm{e}_{1}\right), \ldots, t_{i}\left(b, \mathrm{e}_{i}, \mathrm{e}_{i-1}\right), a, t_{i}\left(a, \mathrm{e}_{i}, \mathrm{e}_{i+1}\right), \ldots, t_{i}\left(a, \mathrm{e}_{i}, \mathrm{e}_{n}\right)\right) \\
& ={ }_{(\text {B2) }} q\left(a, \mathrm{e}_{i}, \ldots, \mathrm{e}_{i}, a, \mathrm{e}_{i}, \ldots, \mathrm{e}_{i}\right) \\
& ={ }_{(\text {B4) }} q\left(a, \mathrm{e}_{i}, \ldots, \mathrm{e}_{i}, q\left(a, \mathrm{e}_{1}, \ldots, \mathrm{e}_{n}\right), \mathrm{e}_{i}, \ldots, \mathrm{e}_{i}\right) \\
& =q\left(a, \mathrm{e}_{i}, \ldots, \mathrm{e}_{i}\right)=\mathrm{e}_{i} .
\end{aligned}
$$

(5): It is easy to check the identity in the generator $\mathbf{n}$ of the variety $n \mathrm{BA}$.

(6): is a consequence of (5).

Theorem 7.13. Let $\phi$ be a congruence, $I(\phi)=\left(\mathrm{e}_{1} / \phi, \ldots, \mathrm{e}_{n} / \phi\right)$ be the multideal of $\mathbf{A}$ determined by $\phi, H=\left(H_{1}, \ldots, H_{n}\right)$ be a multideal and $\theta_{H}$ be the congruence on $\mathbf{A}$ determined by $H$. Then

$$
\theta_{I(\phi)}=\phi \quad \text { and } \quad I\left(\theta_{H}\right)=H .
$$

Proof. We first prove $I\left(\theta_{H}\right)_{k}=H_{k}$, for all $k$. Recall that $\mathrm{e}_{j}=\left(\mathrm{e}_{i}\right)_{\mathrm{i}}$ is the $i$-coordinate of $\mathrm{e}_{i}$ and $\mathrm{e}_{i}=\left(\mathrm{e}_{i}\right)_{\mathrm{k}}$ is the $k$-coordinate of $\mathrm{e}_{i}$ for every $k \neq i$.

(1) First we provide the proof for $k=i$. Let $a \in I\left(\theta_{H}\right)_{i}$. If $a \theta_{H} \mathrm{e}_{i}$ then $f_{H}\left(a_{\mathrm{i}}\right)=$ $f_{H}\left(\left(\mathrm{e}_{i}\right)_{\mathrm{i}}\right)=f_{H}\left(\mathrm{e}_{j}\right)$, that implies $a_{\mathrm{i}} \in H^{*}$. By Lemma 7.10(a) we get the conclusion $a \in H_{i}$.

For the converse, let $a \in H_{i}$. By Lemma 7.10(a) we have $a_{\mathrm{i}} \in H^{*}$ and by Lemma 7.10(b) $a_{\mathrm{k}} \in H_{*}$ for all $k \neq i$. This implies $f_{H}\left(a_{\mathrm{i}}\right)=f_{H}\left(\mathrm{e}_{j}\right)=f_{H}\left(\left(\mathrm{e}_{i}\right)_{\mathrm{i}}\right)$ and $f_{H}\left(a_{\mathrm{k}}\right)=f_{H}\left(\mathrm{e}_{i}\right)=f_{H}\left(\left(\mathrm{e}_{i}\right)_{\mathrm{k}}\right)$ for all $k \neq i$, that implies $a \theta_{H} \mathrm{e}_{i}$. Since $I\left(\theta_{H}\right)_{i}=\mathrm{e}_{i} / \theta_{H}$, we conclude.

(2) Let now $k \neq i$. By Lemma 6.7 we have $H_{k}=H_{i}^{(i k)}$. Let $a \in H_{k}$. Then $a=b^{(i k)}$ for some $b \in H_{i}$. As, by (1), $b \theta_{H} \mathrm{e}_{i}$, then we have $a=b^{(i k)} \theta_{H}\left(\mathrm{e}_{i}\right)^{(i k)}=\mathrm{e}_{k}$. Since $I\left(\theta_{H}\right)_{k}=\mathrm{e}_{k} / \theta_{H}$, we conclude. Now, assuming $a \theta_{H} \mathrm{e}_{k}$, we have: $b=(a)^{(i k)} \theta_{H}\left(\mathrm{e}_{k}\right)^{(i k)}=$ $\mathrm{e}_{i}$. Then $b \in H_{i}$ and $a=b^{(i k)} \in H_{k}$.

Let $\phi$ be a congruence.

(a) Let $a \phi b$. Then $\forall h . a_{\mathrm{h}} \phi b_{\mathrm{h}}$. Since $\phi$ restricted to $\mathbf{B}_{i j}$ is also a Boolean congruence, then we obtain $\left(a_{\mathrm{h}} \oplus_{i j} b_{\mathrm{h}}\right) \phi \mathrm{e}_{i}$, where $\oplus_{i j}$ denotes the symmetric difference in the Boolean centre $\mathbf{B}_{i j}$. We now prove that $a \theta_{I(\phi)} b$. We have $a \theta_{I(\phi)} b$ iff $\forall h . f_{I(\phi)}\left(a_{\mathrm{h}}\right)=f_{I(\phi)}\left(b_{\mathrm{h}}\right)$ iff $\forall h . a_{\mathrm{h}} \oplus_{i j} b_{\mathrm{h}} \in I(\phi)_{*}=B_{i j} \cap \mathrm{e}_{i} / \phi$ iff $\forall h . a_{\mathrm{h}} \oplus_{i j} b_{\mathrm{h}} \in \mathrm{e}_{i} / \phi$ iff $\forall h .\left(a_{\mathrm{h}} \oplus_{i j} b_{\mathrm{h}}\right) \phi \mathrm{e}_{i}$. This last relation is proved above and we conclude $a \theta_{I(\phi)} b$.

(b) Let $a \theta_{I(\phi)} b$. Then $\forall h . a_{\mathrm{h}} \oplus_{i j} b_{\mathrm{h}} \in \mathrm{e}_{i} / \phi$ that implies $\forall h . a_{\mathrm{h}} \phi b_{\mathrm{h}}$, because $\phi$ restricted to $\mathbf{B}_{i j}$ is a Boolean congruence. Since by Lemma 7.12(5) there is a $n$-ary term $u$ such that $a=u\left(a_{1}, \ldots, a_{\mathrm{n}}\right)$ and $b=u\left(b_{1}, \ldots, b_{\mathrm{n}}\right)$, then we conclude $a \phi b$ by using $\forall h . a_{\mathrm{h}} \phi b_{\mathrm{h}}$.

\subsection{Ultramultideals}

In the Boolean case, there is a bijective correspondence between maximal ideals and homomorphisms onto 2 . In this section we show that every multideal can be extended to an ultramultideal, and that there exists a bijective correspondence between ultramultideals and homomorphisms onto $\mathbf{n}$. We also show that prime multideals coincide with ultramultideals.

Let $\left(I_{1}, \ldots, I_{n}\right)$ be a multideal of a $n \mathrm{BA} \mathbf{A}$ and $U$ be a Boolean ultrafilter of $\mathbf{B}_{i j}$ that extends $I^{*}=B_{i j} \cap I_{j}$, and so the maximal ideal $\bar{U}=B_{i j} \backslash U$ extends $I_{*}=B_{i j} \cap I_{i}$. 
Lemma 7.14. For all $a \in A$, there exists a unique $k$ such that $a_{\mathrm{k}} \in U$.

Proof. By Lemma 7.7(ii) the meet of two distinct coordinates is the bottom element $\mathrm{e}_{i}$. Then at most one coordinate may belong to $U$. On the other hand, if all coordinates belong to $\bar{U}$, then the top element $\mathrm{e}_{j}$ belong to $\bar{U}$.

Let $\left(G_{k}\right)_{k \in \hat{n}}$ be the sequence such that $G_{k}=\left\{a \in A: a_{\mathrm{k}} \in U\right\}$, which, by Lemma 7.14, is well defined.

Lemma 7.15. $\left(G_{k}\right)_{k \in \hat{n}}$ is a ultramultideal which extends $\left(I_{k}\right)_{k \in \hat{n}}$.

Proof. (m1): $\mathrm{e}_{k} \in G_{k}$ because $\left(\mathrm{e}_{k}\right)_{\mathrm{k}}=\mathrm{e}_{j} \in U$.

(m2): Let $a \in G_{r}, b \in G_{k}$, and $c_{1}, \ldots, c_{n} \in A$. By Lemma 7.7(ii),

$$
q\left(a, c_{1}, \ldots, c_{r-1}, b, c_{r+1} \ldots, c_{n}\right)_{\mathrm{k}}=\left[\bigvee_{s \neq r}\left(a_{\mathrm{s}} \wedge_{i}\left(c_{s}\right)_{\mathrm{k}}\right)\right] \vee_{i}\left(a_{\mathrm{r}} \wedge_{i} b_{\mathrm{k}}\right)
$$

Since $a_{\mathrm{r}}, b_{\mathrm{k}} \in U$, then $a_{\mathrm{r}} \wedge_{i} b_{\mathrm{k}} \in U$, and so $a_{\mathrm{r}} \wedge_{i} b_{\mathrm{k}} \sqsubseteq\left[\bigvee_{s \neq r}\left(a_{\mathrm{s}} \wedge_{i}\left(c_{s}\right)_{\mathrm{k}}\right)\right] \vee_{i}\left(a_{\mathrm{r}} \wedge_{i} b_{\mathrm{k}}\right) \in U$, where $\sqsubseteq$ is the Boolean order of the Boolean algebra $\mathbf{B}_{i j}$. Hence,

$$
q\left(a, c_{1}, \ldots, c_{r-1}, b, c_{r+1} \ldots, c_{n}\right) \in G_{k} .
$$

(m3): It can be proved similarly.

We now prove that $\left(G_{k}\right)_{k \in \hat{n}}$ extends $\left(I_{k}\right)_{k \in \hat{n}}$. It is sufficient to show that, for every $a \in I_{k}$, we have that $a_{\mathrm{k}} \in U$. We get the conclusion by Lemma 7.10(a).

\section{Theorem 7.16.}

(i) Every multideal can be extended to an ultramultideal.

(ii) There is a bijective correspondence between ulramultideals and homomorphisms onto $\mathbf{n}$.

Proof. (i) follows from Lemma 7.15. Regarding (ii), we remark that the algebra $\mathbf{n}$ is the unique simple $n \mathrm{BA}$.

We conclude this section by characterising prime multideals.

Definition 7.17. We say that a multideal $\left(I_{1}, \ldots, I_{n}\right)$ is prime if, for every $i, a \wedge_{i} b \in I_{i}$ implies $a \in I_{i}$ or $b \in I_{i}$.

Proposition 7.18. A multideal is prime iff it is an ultramultideal.

Proof. $(\Rightarrow)$ : Let $\left(I_{1}, \ldots, I_{n}\right)$ be a prime multideal. If $a \in B_{i j}$, then $a \wedge_{i} \neg_{i j}(a)=\mathrm{e}_{i} \in I_{i}$. Then either $a$ or $\neg_{i j}(a) \in I_{i}$. This implies that $I_{*}=B_{i j} \cap I_{i}$ is a maximal Boolean ideal and the complement $I^{*}=B_{i j} \cap I_{j}$ is a Boolean ultrafilter.

Let now $b \in A$ such that $b \notin I=\bigcup_{k=1}^{n} I_{k}$. By Lemma 7.10(a) we have that $b \in I_{r}$ iff $b_{\mathrm{r}} \in I^{*}$. Then $b_{\mathrm{r}} \notin I^{*}$ for all $r$. Since $I^{*}$ is a Boolean ultrafilter, then $b_{\mathrm{r}} \in I_{*}$ for all $r$. Hence $\mathrm{e}_{j}=\bigvee_{r=1}^{n} b_{\mathrm{r}} \in I_{*}$, contradicting the fact that the top element does not belong to a maximal ideal. In conclusion, $b \in I=\bigcup_{k=1}^{n} I_{k}$ for an arbitrary $b$, so that $I=A$.

$(\Leftarrow)$ : Let $I$ be an ultramultideal. Let $a \wedge_{i} b \in I_{i}$ with $a \in I_{r}$ and $b \in I_{k}$ (with $r \neq i$ and $k \neq i)$. Then by property (m2) of multideals we get $a \wedge_{i} b=t_{i}\left(a, b, \mathrm{e}_{i}\right)=$ $q\left(a, b, \ldots, b, \mathrm{e}_{i}, b, \ldots, b\right) \in I_{k}$. Contradiction. 


\section{Conclusion}

Boolean-like algebras have been introduced in $[6,23]$ as a generalisation of Boolean algebras to any finite number of truth values. Boolean-like algebras provide a new characterisation of primal varieties exhibiting a perfect symmetry of the values of the generator of the variety. In this paper we have investigated the relationships between skew Boolean algebras and Boolean-like algebras. We have shown that any $n$-dimensional Boolean-like algebra is a skew cluster of $n$ isomorphic right-handed skew Boolean algebras, and that the variety of skew star algebras is term equivalent to the variety of Boolean-like algebras. Moreover, we have got a representation theorem for right-handed skew Boolean algebras, and developed a general theory of multideals for Boolean-like algebras. Several further topics are worth mentioning:

1. How is the duality theory of SBAs and BAs related to a possible duality theory of $n$ BAs (a Stone-like topology on ultramultideals).

2. Find a more satisfactory axiomatisation of skew star algebras.

3. Each SBA living inside a $n \mathrm{BA}$ has a bottom element 0 and several maximal elements. The construction could be made symmetric, by defining "skew-like" algebras having several minimal and several maximal elements.

4. For each $n \mathrm{BA} \mathbf{A}$, the algebras $S_{1}(\mathbf{A}), \ldots, S_{n}(\mathbf{A})$, constituting the skew cluster of $\mathbf{A}$, are isomorphic. This result is also of technical interest for the following open problem in the theory of skew Boolean algebras:

Problem 7.19. Given a SBA A with a maximal class $M \supseteq\left\{m_{1}, m_{2}\right\}$, let $\mathbf{A}_{m_{1}}$ and $\mathbf{A}_{m_{2}}$ be the algebras obtained from $\mathbf{A}$ on distinguishing the elements $m_{1}$ and $m_{2}$ respectively. Are the algebras $\mathbf{A}_{m_{1}}$ and $\mathbf{A}_{m_{2}}$ isomorphic?

This problem is part of the folklore and it does not appear in any published work to date. It is implicit in Leech [17], where both skew Boolean algebras (as they are now understood) and skew Boolean algebras possessing a maximal class are introduced.

The difficulty in obtaining a solution to Problem 7.19 evidently lies in constructing the required isomorphism. For skew Boolean $\cap$-algebras, a related problem has been considered and resolved in the positive by Bignall [2]. The proof exploits sheaf (Boolean product) representations to obtain the desired isomorphism; as skew Boolean algebras admit only a weak Boolean product representation, the proof does not seem readily adaptable.

Problem 7.19 is of purely technical interest in the theory of skew Boolean algebras. However, it assumes greater prominence in logics arising from (structurally enriched) skew Boolean algebras. Very roughly speaking, let $S$ be an algebraisable logic arising from a quasivariety $K$ of 1-regular (necessarily structurally enriched) skew Boolean algebras. Given $n$ residually distinct constant terms of $K, 1<n<\omega$ (working with the finite case for simplicity), $S$ admits $n-1$ negation connectives via implication into $m$, for each $m$ a constant term distinct from 1. A positive solution to Problem 7.19 would imply that these $n-1$ negations are not essentially different, and hence that it is enough to fix a single such negation univocally when studying $S$; whereas a negative solution to Problem 7.19 would imply that these $n-1$ negations are all distinct, and hence that they must all be accounted for in any study of $S$. 


\section{References}

[1] A. Bauer and K. Cvetko-Vah, Stone duality for skew Boolean algebras with intersections, Houston J. Math. 39 (2013), 73-109.

[2] R. J. Bignall, Quasiprimal Varieties and Components of Universal Algebras, Ph.D. thesis, The Flinders University of South Australia, Australia, 1976.

[3] R. J. Bignall and J. E. Leech, Skew Boolean algebras and discriminator varieties, Algebra Universalis 33 (1995), 387-398, doi:10.1007/bf01190707.

[4] R. J. Bignall and M. Spinks, Dual binary discriminator varieties, Art Discrete Appl. Math. 2 (2019), \#P2.08, doi:10.26493/2590-9770.1324.5b2.

[5] W. J. Blok and D. Pigozzi, On the structure of varieties with equationally definable principal congruences III, Algebra Universalis 32 (1994), 545-608, doi:10.1007/bf01195727.

[6] A. Bucciarelli, A. Ledda, F. Paoli and A. Salibra, Boolean-like algebras of finite dimension, 2018, preprint, arXiv:1806.06537 [CS.LO].

[7] S. Burris and H. P. Sankappanavar, A Course in Universal Algebra, volume 78 of Graduate Texts in Mathematics, Springer-Verlag, New York, 1981, http://www.math. uwaterloo.ca/ snburris/htdocs/ualg.html.

[8] K. Cvetko-Vah, J. Leech and M. Spinks, Skew lattices and binary operations on functions, $J$. Appl. Log. 11 (2013), 253-265, doi:10.1016/j.jal.2013.03.011.

[9] K. Cvetko-Vah and A. Salibra, The connection of skew Boolean algebras and discriminator varieties to Church algebras, Algebra Universalis 73 (2015), 369-390, doi:10.1007/ s00012-015-0320-9.

[10] K. Fichtner, Eine Bemerkung über Mannigfaltigkeiten universeller Algebren mit Idealen, Monatsb. Deutsch. Akad. Wiss. Berlin 12 (1970), 21-25.

[11] S. Gudder, Boolean vector spaces, 2014, preprint.

[12] S. Gudder and F. Latrémolière, Boolean inner-product spaces and Boolean matrices, Linear Algebra Appl. 431 (2009), 274-296, doi:10.1016/j.laa.2009.02.028.

[13] K. Iséki and S. Tanaka, An introduction to the theory of BCK-algebras, Math. Japon. 23 (1978/79), 1-26.

[14] G. Kudryavtseva, A refinement of Stone duality to skew Boolean algebras, Algebra Universalis 67 (2012), 397-416, doi:10.1007/s00012-012-0192-1.

[15] A. Ledda, F. Paoli and A. Salibra, On semi-Boolean-like algebras, Acta Univ. Palack. Olomuc. Fac. Rerum Natur. Math. 52 (2013), 101-120.

[16] J. Leech, Skew lattices in rings, Algebra Universalis 26 (1989), 48-72, doi:10.1007/ bf01243872.

[17] J. Leech, Skew Boolean algebras, Algebra Universalis 27 (1990), 497-506, doi:10.1007/ bf01188995.

[18] J. Leech, Recent developments in the theory of skew lattices, Semigroup Forum 52 (1996), 7-24, doi:10.1007/bf02574077.

[19] J. Leech and M. Spinks, Skew Boolean algebras derived from generalized Boolean algebras, Algebra Universalis 58 (2008), 287-302, doi:10.1007/s00012-008-2069-x.

[20] G. Manzonetto and A. Salibra, From lambda-calculus to universal algebra and back, in: E. Ochmanski and J. Tyszkiewicz (eds.), Mathematical Foundations of Computer Science 2008, Springer, volume 5162 of Lecture Notes in Computer Science, 2008 pp. 479-490, doi: 10.1007/978-3-540-85238-4\_39, proceedings of thee 33rd International Symposium, MFCS 2008 held in Torun, Poland, August 25 - 29, 2008. 
[21] R. N. McKenzie, G. F. McNulty and W. F. Taylor, Algebras, Lattices, Varieties, Volume I, The Wadsworth \& Brooks/Cole Mathematics Series, Wadsworth \& Brooks/Cole Advanced Books \& Software, Monterey, California, 1987.

[22] A. Salibra, A. Ledda and F. Paoli, Boolean product representations of algebras via binary polynomials, in: J. Czelakowski (ed.), Don Pigozzi on Abstract Algebraic Logic, Universal Algebra, and Computer Science, Springer, Cham, volume 16 of Outstanding Contributions to Logic, pp. 297-321, 2018, doi:10.1007/978-3-319-74772-9_12.

[23] A. Salibra, A. Ledda, F. Paoli and T. Kowalski, Boolean-like algebras, Algebra Universalis 69 (2013), 113-138, doi:10.1007/s00012-013-0223-6.

[24] B. M. Schein, Bands of semigroups: variations on a Clifford theme, in: K. H. Hofmann and M. W. Mislove (eds.), Semigroup Theory and Its Applications, Cambridge University Press, Cambridge, volume 231 of London Mathematical Society Lecture Note Series, 1996 pp. 5380, doi:10.1017/cbo9780511661877.006, proceedings of the conference commemorating the work of Alfred H. Clifford held at Tulane University, New Orleans, LA, March 1994.

[25] M. Spinks, Automated Deduction in Non-Commutative Lattice Theory, Technical Report 3/98, 1998.

[26] M. Spinks, Contributions to the Theory of Pre-BCK-Algebras, Ph.D. thesis, Monash University, Australia, 2003, https://monash.figshare.com/articles/Contributions_ to_the_theory_of_pre-BCK-algebras/5446369.

[27] D. Vaggione, Varieties in which the Pierce stalks are directly indecomposable, J. Algebra 184 (1996), 424-434, doi:10.1006/jabr.1996.0268.

[28] H. Werner, Discriminator-Algebras: Algebraic Representation and Model Theoretic Properties, volume 6 of Studien zur Algebra und ihre Anwendungen, Akademie-Verlag, Berlin, 1978. 